\title{
Hamiltonian and Lagrangian Dynamical Matrix Approaches Applied to Magnetic Nanostructures
}

\author{
Roberto Zivieri ${ }^{1}$ and Giancarlo Consolo² \\ ${ }^{1}$ CNISM Unit of Ferrara and Department of Physics, University of Ferrara, Via Saragat 1, 44122 Ferrara, Italy \\ ${ }^{2}$ Department of Sciences for Engineering and Architecture, University of Messina, C.da di Dio, Villaggio S.Agata, \\ 98166 Messina, Italy
}

Correspondence should be addressed to Roberto Zivieri, zivieri@fe.infn.it

Received 30 March 2012; Accepted 23 April 2012

Academic Editor: Eduardo Martinez Vecino

Copyright (C) 2012 R. Zivieri and G. Consolo. This is an open access article distributed under the Creative Commons Attribution License, which permits unrestricted use, distribution, and reproduction in any medium, provided the original work is properly cited.

Two micromagnetic tools to study the spin dynamics are reviewed. Both approaches are based upon the so-called dynamical matrix method, a hybrid micromagnetic framework used to investigate the spin-wave normal modes of confined magnetic systems. The approach which was formulated first is the Hamiltonian-based dynamical matrix method. This method, used to investigate dynamic magnetic properties of conservative systems, was originally developed for studying spin excitations in isolated magnetic nanoparticles and it has been recently generalized to study the dynamics of periodic magnetic nanoparticles. The other one, the Lagrangian-based dynamical matrix method, was formulated as an extension of the previous one in order to include also dissipative effects. Such dissipative phenomena are associated not only to intrinsic but also to extrinsic damping caused by injection of a spin current in the form of spin-transfer torque. This method is very accurate in identifying spin modes that become unstable under the action of a spin current. The analytical development of the system of the linearized equations of motion leads to a complex generalized Hermitian eigenvalue problem in the Hamiltonian dynamical matrix method and to a non-Hermitian one in the Lagrangian approach. In both cases, such systems have to be solved numerically.

\section{Introduction}

In these last years, great attention has been given to the study of magnetization dynamics in laterally confined magnetic systems. It is well know that spin excitations are quantized due to the lateral confinement. The oscillations are the socalled normal modes, which represent a pattern of motion given by all the parts of the system oscillating sinusoidally with the same frequency and with the same phase relation. In this last decade, analytical models have given important contributions to understand the frequency spectrum of normal modes for different ground-state magnetizations [111]. However, some limitations due to the assumptions made for the determination of the equilibrium state, the boundary conditions, and the calculation of the energy contributions to normal modes dynamics are still present.

On the other hand, a lot of efforts have been devoted to develop micromagnetic codes having the aim of calculating very precisely different ground states of nanometric particles [12]. Due to their accuracy, the developed micromagnetic methods have contributed to give additional information about the spin dynamics. The first micromagnetic calculations were typically based upon codes developed to calculate in the first place the ground state of a given magnetic particle. Then the time evolution of the average magnetization of a particle could be obtained and, from a subsequent postprocessing of these data (mainly using the Fourier transform of the magnetization), information could be extracted about mode frequencies and spatial profiles [13]. In the simplest application of the method, the limit of these calculations was the observation of modes with nonzero magnetization only. More recently, a micromagnetic method was extended to the detailed calculation of eigenfrequencies and eigenvectors under the effect of an oscillatory in-plane small magnetic field [14]. Another recent micromagnetic method was also developed to study the quantized spin excitations in laterally 
confined systems [15]. This is the so-called Hamiltonianbased dynamical matrix method (HDMM).

The HDMM was first formulated for isolated magnetic nanoelements and then it has been generalized to the case of interacting nanoelements [16]. It is the prototype of finitedifference methods and represents an eigenvalue/eigenvector problem. The scope of this method is to find the frequencies and profiles of the spin modes which are associated to the eigenvalues and the eigenvectors, respectively, of a dynamical matrix. It can be considered the analogous of the dynamical matrix formalism used to find atomic vibrations (phonons) in crystalline solids. The dynamical matrix contains the second derivatives of the density energy coming from a second order expansion of the density energy around the equilibrium. This method was already used to study the spin excitations in magnetic multilayers with ferro- or antiferromagnetic coupling $[17,18]$. Due to the translational invariance, the number of independent dynamic variables was reduced to twice the number of the layers. The calculated second derivatives are evaluated at equilibrium. The eigenvalue/eigenvector problem can be set as a complex generalized Hermitian eigenvalue problem. The method presents several advantages: a single calculation yields the frequencies and eigenvectors of all modes of any symmetry, it is applicable to a particle of any shape (within the nanometric range), and the computation time is affordable. This means that by means of this micromagnetic approach, it is possible to determine, after a single iteration, the frequencies and the profiles of all spin-modes, independently of the ground-state magnetization (e.g., vortex state, vortex in the presence of an external magnetic field, onion state, quasi-saturated state). The main restriction of the method is its applicability to confined magnetic systems whose spin dynamics is assumed purely precessional with no dissipative effects. Of course, this is true only in a first approximation, since in real magnetic systems the intrinsic damping process plays an important rule. In order to select the representative modes of the spectrum and to compare them with the ones observed by means of the experimental techniques, the differential scattering cross-section has to be evaluated both for noninteracting and interacting magnetic particles.

The first applications of the HDMM were on chains of dipolarly interacting rectangular dots representing a onedimensional array [19] and of two-dimensional (2D) arrays formed by circular nanometric disks [20]. Very recently, this method was applied to study the collective mode dynamics in arrays of holes embedded into a thin ferromagnetic film. This calculation was done by including in the energy density computation also the exchange interaction between micromagnetic cells belonging to two adjacent primitive cells [21].

In order to overcome the above-mentioned restrictions of the HDMM, Consolo et al. formulated very recently the so-called Lagrangian-based dynamical matrix method (LDMM) [22]. Such a method explicitly takes into account the intrinsic "positive" Gilbert damping and the currentinduced spin-transfer-torque "negative" dissipation. Since the magnetic system so obtained is no more conservative, a Lagrangian formalism is necessary. Unlike the HDMM,

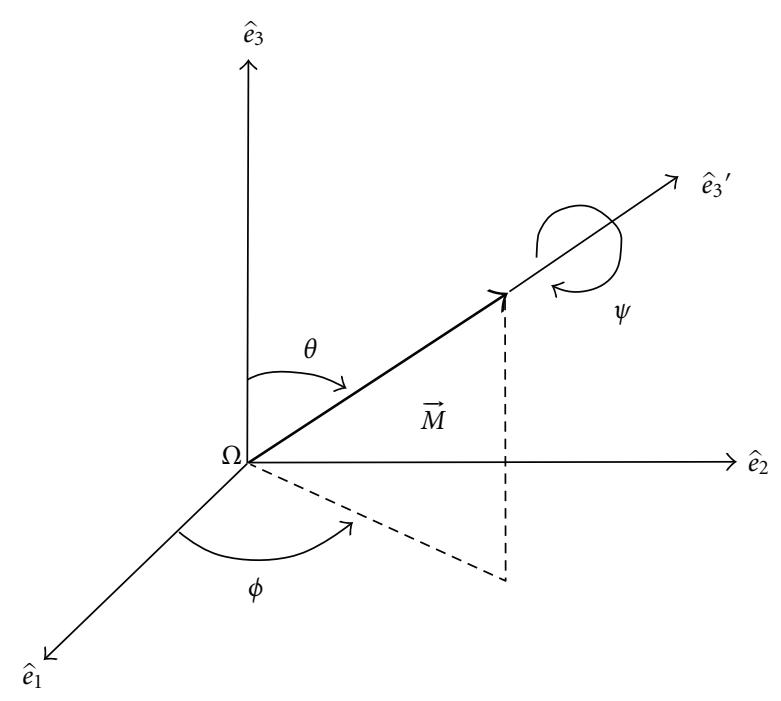

FIGURE 1: Reference frame used in the theory.

the LDMM cannot be cast as a classical (nongeneralized) eigenvalue/eigenvector problem, but it has to be formulated as a complex generalized non-Hermitian eigenvalue problem. The first application of the method was done on a magnetic nanopillar stack of circular cross-section subject to an external magnetic field directed a few degree away from the normal to the plane [22]. The analysis was then extended to the case of external magnetic fields of variable intensity and orientation with respect to the plane of the nanopillar [23].

It is important to notice that both formalisms have been developed up to now in the linear approximation, namely, considering small angular deviations of the magnetization from equilibrium so that each spin excitation is a normal mode of the system.

The reference frame used in the micromagnetic calculations performed both by means of HDMM and LDMM is illustrated in Figure 1. The $z$-axis is along the normal to the particle and the $x-y$ plane lies on the particle plane. According to this reference frame, the configuration of the vector $\vec{M}$, representative of the magnetic dipole momentum, is identified through the polar angles, $\theta$ and $\phi$, and the intrinsic rotation $\psi$. As it will appear clear in the section devoted to the Lagrangian approach, this latter angle does not enter in the equation of motion, being the corresponding Lagrange equation associated to a first integral of the motion (the conservation of the angular momentum). So that, as it is expected, because the modulus of the magnetization vector is preserved in time, the dynamics can be described through two degrees of freedom only (typically $\theta$ and $\phi$ ).

If we assume that the magnetization vector $\vec{M}$ is placed along the generic direction given by the unitary vector $\hat{e}_{3}^{\prime}$, it can be expressed in Cartesian coordinates by

$$
\vec{M}(t)=M_{s}(\sin \theta(t) \cos \phi(t), \sin \theta(t) \sin \phi(t), \cos \theta(t)),
$$


being $M_{s}$ the saturation magnetization value (the modulus of the magnetization vector) and the time dependence of the angles $\theta$ and $\phi$ expressed as

$$
\begin{aligned}
& \theta(t)=\Theta+\delta \theta(t), \\
& \phi(t)=\Phi+\delta \phi(t) .
\end{aligned}
$$

In (2), we indicate by $(\Theta, \Phi)$ the static (equilibrium) orientation of the magnetization obtained by solving the stationary problem $(\dot{\vec{M}}=0)$ for whatever effective field and magnetization distributions. Instead, $\delta \theta(t)$ and $\delta \phi(t)$ are the small polar and azimuthal deviations from equilibrium, respectively.

In the micromagnetic calculations, the magnetic system is subdivided into rectangular cells. Each micromagnetic cell is identified by a single index $k(j)$ that varies from 1 to $N$, where $N$ denotes the number of cells. Hence, $M_{k}$ is the magnetization in the $k$ th cell and $\vec{r}_{i j}=\vec{r}_{i}-\vec{r}_{j}$ is the in-plane distance between the $i$ th cell and the $j$ th cell. The index has been assigned so that the first line of the rectangular matrix $(X \times Y)$ corresponds to $k=1, \ldots, Y$ and the second line to $k=Y+1, \ldots, 2 Y$, and so on and so forth. $X(Y)$ is the number of cells along $x(y)$, while $Z$ is the number of cells along $z$ for a sample of thickness equal to $d$. We define for each cell the reduced magnetization $\vec{m}_{k}=\vec{M}_{k} / M_{s}$. Hence, in a polar reference frame for each cell

$$
\vec{m}_{k}(t)=\left(\sin \theta_{k}(t) \cos \phi_{k}(t), \sin \theta_{k}(t) \sin \phi_{k}(t), \cos \theta_{k}(t)\right),
$$

where $\phi_{k}$ is the azimuthal angle and $\theta_{k}$ is the polar angle of the magnetization. The total energy density of the system, obtained by dividing the total energy by the volume of the cell, is a function of $\phi_{k}$ and $\theta_{k}: E=E\left(\theta_{k}, \phi_{k}\right)$ where $k$ varies from 1 to $N$.

In the following sections we describe in detail the two micromagnetic methods. We do not illustrate the applications of these methods to magnetic nanoparticles, because this aspect is not the purpose of this paper. We give a summary of the paper. In Section 2, we outline the formalism at the basis of the HDMM. First, we introduce the different contributions to the energy density and then we derive the system of linear and homogeneous equations of motion for the isolated particle from the Hamilton equations. Finally, we present the generalization of the HDMM to the case of interacting elements. In Section 3, the formalism at the basis of the LDMM is presented. First, the Lagrangian equations for a macrospin system are derived. Then a generalization is given by considering $N$ interacting momenta in an isolated magnetic element.

\section{HDMM}

This section deals with the review of the HDMM formalism. As stated above, the HDMM is a micromagnetic approach that can be applied only to fully conservative systems which are supposed to have, in a first approximation, a purely (undamped) precessional motion of the magnetization about the effective field. It is a finite-difference micromagnetic method developed in the linear regime of spin dynamics by considering small deviations from the equilibrium magnetization. In the first subsection, the different contributions of the energy density entering into the dynamical matrix are calculated and the equations of motion for an isolated magnetic element are cast in the form of a linear and homogeneous system that can be solved as a complex generalized Hermitian eigenvalue problem. In the last subsection, it is shown the generalization of the HDMM to interacting magnetic nanoparticles by including into the dynamical matrix the Bloch condition.

2.1. Energy Density. First, we give the explicit expressions of the different interactions entering into the total micromagnetic energy density $E$ of a given confined magnetic system simulated by using the HDMM: Zeeman energy, exchange energy, demagnetizing energy, and anisotropy energy, respectively [24]. The energy density is defined as $E=\widetilde{E} / V$ where $\widetilde{E}$ is the energy of the system and $V$ its volume. In the presence of an external magnetic field $\vec{H}$, the Zeeman energy density can be written in the form

$$
E_{\mathrm{ext}}=-\mu_{0} M_{s} \vec{H} \cdot \sum_{k=1}^{N} \vec{m}_{i}
$$

being $\mu_{0}$ the vacuum permeability. In micromagnetic theory, the exchange energy can be expressed as a volume integral of the form

$$
\widetilde{E}_{\text {exch }}=A \int_{\text {part }} \sum_{j=1}^{3}\left(\vec{\nabla} m_{j}\right)^{2} d V,
$$

where the subscript "part" denotes the volume of a general magnetic particle, $A$ is the exchange stiffness constant, and $\vec{\nabla}$ is the gradient applied to a given component of the magnetization. In this case the exchange contribution is independent of $z$. Using the first-neighbours model, the exchange energy density can be written as follows:

$$
E_{\text {exch }}=A \sum_{k=1}^{N} \sum_{n=1}^{4} \frac{1-\vec{m}_{k} \cdot \vec{m}_{n}}{a_{k n}^{2}}
$$

where the variable $a_{k n}$ is the distance between the centers of two adjacent cells of index $k$ and $n$, respectively, $k$ varies over all micromagnetic cells, and the sum over $n$ ranges over the neighbours of the $k$-th cell. If the micromagnetic cells $k$ are situated at the edges, one must impose boundary conditions. The cells on the edges interact with an external row of cells that have the same fixed magnetization, in this case the corresponding term in the sum must be weighted twice.

In order to calculate the demagnetizing energy density, we have followed the method of the demagnetizing tensor. In the following, we use also the term dipolar in place of the term demagnetizing, because the higher-order terms of the expansion vanish in the practical cases examined. Generally, within the framework of the demagnetizing tensor 
method, the demagnetizing energy density can be written as follows:

$$
\begin{aligned}
E_{\mathrm{dmg}} & =\frac{1}{2} \mu_{0} \sum_{k j} \vec{M}_{k} \cdot \underline{\underline{N}} \vec{M}_{j} \\
& =\mu_{0} \frac{M_{s}^{2}}{2} \sum_{k j}\left(m_{x k}, m_{y k}, m_{z k}\right) \times\left(\begin{array}{lll}
N_{x x} & N_{x y} & N_{x z} \\
N_{y x} & N_{y y} & N_{y z} \\
N_{z x} & N_{z y} & N_{z z}
\end{array}\right)\left(\begin{array}{l}
m_{x j} \\
m_{y j} \\
m_{z j}
\end{array}\right) .
\end{aligned}
$$

This equation includes the self-energy and $N_{\alpha \beta}=N_{\alpha \beta}\left(\vec{r}_{k j}\right)$ with $\alpha, \beta=x, y, z$ are the elements of demagnetizing tensor. Each component of the demagnetizing tensor is related to the interaction between two rectangular surfaces $S$ and $S^{\prime}$. Under the assumption made for the calculation of the demagnetizing field for uniform magnetization, by using a version of Gauss's theorem, the demagnetizing tensor can be written as

$$
\underline{\underline{N}}\left(\vec{r}_{k j}\right)=\frac{1}{V} \int_{S_{k}} d \vec{S} \int_{S_{j}^{\prime}} \frac{d \overrightarrow{S^{\prime}}}{\left|\vec{r}-\vec{r}^{\prime}\right|},
$$

where $V=l_{c}^{2} d$ is the volume of the micromagnetic cell with $l_{c}$ the cell size and $d$ is the cell height. Because of the fourfold $C_{4}$ symmetry and since $\vec{r}_{k j} \equiv(x, y, z)$, all components can be expressed only as a function of $N_{x x}(x, y, z)$ and $N_{x y}(x, y, z)$ components with suitable permutations of the variables $x, y, z$.

The magnetocrystalline uniaxial anisotropy can be labeled with the symbol $E_{\text {ani }}$. It is an energy density function, for a given micromagnetic cell, of the angle $\alpha_{k}$ between the magnetization of the single cell $\vec{m}_{k}$ and the easy axis of generic direction given by the unit vector $\hat{u}$. We write

$$
\begin{aligned}
E_{\mathrm{ani}} & =\sum_{k=1}^{N} K^{(1)} \sin ^{2} \alpha_{k}=\sum_{k=1}^{N} K^{(1)}\left(1-\cos ^{2} \alpha_{k}\right) \\
& =\sum_{k=1}^{N} K^{(1)}\left[1-\left(\vec{m}_{k} \cdot \hat{u}\right)^{2}\right],
\end{aligned}
$$

where $K^{(1)}$ is the first-order anisotropy uniaxial coefficient.

As the dynamical matrix components are expressed in terms of the second derivatives of the energy density, it is necessary to calculate them from the above expressions. First, we calculate the second derivatives of the magnetization with respect to the polar and azimuthal angles of the given micromagnetic cell that represent the degrees of freedom of the system. Indeed, the second derivatives of the magnetization appear in the final expressions of the second derivatives of the energy density. In particular

$$
\begin{gathered}
\frac{\partial^{2} \vec{m}_{k}}{\partial \delta \phi_{k}^{2}}=\left(-\sin \theta_{k} \cos \phi_{k},-\sin \theta_{k} \sin \phi_{k}, 0\right), \\
\frac{\partial^{2} \vec{m}_{k}}{\partial \delta \phi_{k} \partial \delta \theta_{k}}=\left(-\cos \theta_{k} \sin \phi_{k}, \cos \theta_{k} \cos \phi_{k}, 0\right), \\
\frac{\partial^{2} \vec{m}_{k}}{\partial \delta \theta_{k}^{2}}=\left(-\sin \theta_{k} \cos \phi_{k},-\sin \theta_{k} \sin \phi_{k},-\cos \theta_{k}\right) .
\end{gathered}
$$

The second derivatives of the energy density are calculated at equilibrium. For the sake of simplicity, in the following, the derivatives are calculated with respect to $\delta \theta_{k}$ and $\delta \theta_{l}$ implying that one or two of the two generic variables could be also $\delta \phi$.

The second derivative of Zeeman energy density becomes

$$
\frac{\partial^{2} E_{\text {ext }}}{\partial \delta \theta_{k} \partial \delta \theta_{l}}= \begin{cases}-\mu_{0} M_{s} \vec{H} \cdot \frac{\partial^{2} \vec{m}_{k}}{\partial \delta \theta_{k} \partial \delta \theta_{l}} & l=k \\ 0 & l \neq k\end{cases}
$$

As outlined previously, for the calculation of the exchange contribution, the nearest-neighbour model is taken into account. It is useful to give also the expression of the first derivative due to some important manipulations that have to be performed. The first derivative with respect to $\delta \theta_{k}$ includes in the sum a term in which $i=k$ and thus $n \neq k$ and also the other terms with $n=k$ and with $i$ one of the nearest neighbours. Thanks to a proper change of indices in the second term, the following equation is obtained:

$$
\begin{aligned}
\frac{\partial E_{\text {exch }}}{\partial \delta \theta_{k}} & =-A \sum_{n=1}^{4} \frac{1}{a_{k n}^{2}} \frac{\partial \vec{m}_{k}}{\partial \delta \theta_{k}} \cdot \vec{m}_{n}-A \sum_{n=1}^{4} \frac{1}{a_{k n}^{2}} \vec{m}_{n} \cdot \frac{\partial \vec{m}_{k}}{\partial \delta \theta_{k}} \\
& =-2 A \sum_{n=1}^{4} \frac{1}{a_{k n}^{2}} \frac{\partial \vec{m}_{k}}{\partial \delta \theta_{k}} \cdot \vec{m}_{n},
\end{aligned}
$$

where the sum over $n$ is made up over the nearest-neighbour micromagnetic cells of the $k$-th cell. In the special case of the adopted first neighbours model, the second derivatives are

$$
\begin{aligned}
& \frac{\partial^{2} E_{\text {exch }}}{\partial \delta \theta_{k} \partial \delta \theta_{l}} \\
& =\left\{\begin{array}{lc}
-2 A \sum_{n=1}^{4} \frac{1}{a_{k n}^{2}} \frac{\partial^{2} \vec{m}_{k}}{\partial \delta \theta_{k} \partial \delta \theta_{l}} \cdot \vec{m}_{n} & l=k \\
-2 A \frac{1}{a_{k l}^{2}} \frac{\partial \vec{m}_{k}}{\partial \delta \theta_{k}} \cdot \frac{\partial \vec{m}_{l}}{\partial \delta \theta_{l}} & l, k: \text { nearest-neighbour } \\
0 & k \neq l \text { and }
\end{array}\right. \\
&
\end{aligned}
$$

We now pass to the calculation of the derivatives of the demagnetizing energy density. Due to their rather complicated form, we give in the following the expression not only of the second derivatives of the demagnetizing energy density but also of the first derivatives with respect to the generic 
variable $\delta \theta_{i}$. The first derivative of the demagnetizing energy density (7) is

$$
\begin{gathered}
\frac{\partial E_{\mathrm{dmg}}}{\partial \delta \theta_{i}}=\mu_{0} M_{s}^{2}\left[\frac{1}{2} \sum_{k \neq i} \vec{m}_{k} \cdot \underline{\underline{N}}(k, i) \frac{\partial \vec{m}_{i}}{\partial \delta \theta_{i}}+\frac{1}{2} \sum_{j \neq i} \frac{\partial \vec{m}_{i}}{\partial \delta \theta_{i}}\right. \\
\left.\cdot \underline{\underline{N}}(k, i) \vec{m}_{j}+\frac{1}{2} \frac{\partial}{\partial \delta \theta_{i}}\left(\vec{m}_{i} \cdot \hat{N}(i, i) \vec{m}_{i}\right)\right] \\
=\mu_{0} M_{s}^{2} \sum_{\substack{k=1 \\
k \neq i}}^{N} \vec{m}_{k} \cdot \underline{\underline{N}}(k, i) \frac{\partial \vec{m}_{i}}{\partial \delta \theta_{i}}+\vec{m}_{i} \cdot \underline{\underline{N}}(i, i) \frac{\partial \vec{m}_{i}}{\partial \delta \theta_{i}}
\end{gathered}
$$

In the sum, the contribution of the terms with the same index $k=j=i$ has been separated; moreover, we have taken into account that the tensor $\underline{\underline{N}}$ fulfils $\underline{\underline{N}}(k, i)=\underline{\underline{N}}(i, k)$ and is symmetric $\left(N_{\alpha \beta}=N_{\beta \alpha}\right)$. write

Thanks to the previous consideration, it is possible to

$$
\frac{\partial \vec{m}_{k}}{\partial \delta \theta_{k}} \cdot \underline{\underline{N}}(k, i) \vec{m}_{i}=\vec{m}_{i} \cdot \underline{\underline{N}}(k, i) \frac{\partial \vec{m}_{k}}{\partial \delta \theta_{k}},
$$

and therefore,

$$
\begin{aligned}
\frac{\partial}{\partial \delta \theta_{i}}\left(\vec{m}_{i} \cdot \underline{\underline{N}}(i, i) \vec{m}_{i}\right) & =\frac{\partial \vec{m}_{i}}{\partial \delta \theta_{i}} \cdot \underline{\underline{N}}(i, i) \vec{m}_{i}+\vec{m}_{i} \cdot \underline{\underline{N}}(i, i) \frac{\partial \vec{m}_{i}}{\partial \delta \theta_{i}} \\
& =2 \vec{m}_{i} \cdot \underline{\underline{N}}(i, i) \frac{\partial \vec{m}_{i}}{\partial \delta \theta_{i}} .
\end{aligned}
$$

The second derivative must take into account the two cases: $i \neq l, i=l(j=i)$, namely,

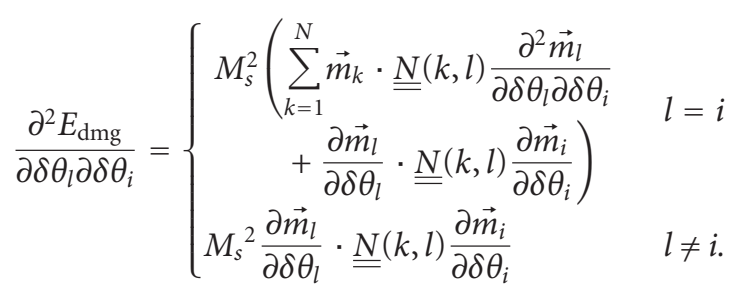

The $k=i$ term resulting from the derivative of the second term (in the expression of the first derivative given above) has been included in the sum over $k$.
The last step is the calculation of the term associated with the anisotropy energy density. The second derivative of the anisotropy energy density can be written as

$$
\begin{aligned}
& \frac{\partial^{2} E_{\text {ani }}}{\partial \delta \phi_{j} \partial \delta \theta_{k}} \\
& =\left\{\begin{array}{r}
-2 K^{(1)}\left[\left(\frac{\partial \vec{m}_{k}}{\partial \delta \phi_{j}} \cdot \hat{u}\right) \cdot\left(\frac{\partial \vec{m}_{k}}{\partial \delta \theta_{k}} \cdot \hat{u}\right)+\left(\vec{m}_{k} \cdot \hat{u}\right)\right. \\
\left.\cdot\left(\frac{\partial^{2} \vec{m}_{k}}{\partial \delta \phi_{j} \partial \delta \theta_{k}} \cdot \hat{u}\right)\right] \quad \text { for } \delta \theta, \delta \phi k=j \\
-2 K^{(1)}\left[\left(\frac{\partial \vec{m}_{k}}{\partial \delta \phi_{k}} \cdot \hat{u}\right)^{2}+\left(\vec{m}_{k} \cdot \hat{u}\right) \cdot\left(\frac{\partial^{2} \vec{m}_{k}}{\partial \delta \phi_{k}^{2}} \cdot \hat{u}\right)\right] \\
\text { for } \delta \phi, \delta \phi \text { or } \delta \theta, \delta \theta k=j \\
k \neq j .
\end{array}\right.
\end{aligned}
$$

2.2. Equations of Motion for an Isolated Magnetic Particle. The derivatives calculated previously are included into the dynamic equations. Indeed, the equations of motion can be cast into a linear and homogeneous system in which the second derivatives of the energy density calculated at equilibrium appear explicitly. It is well know that the equation of motion for a magnetic spin system which undergoes a purely precessional motion is the Landau-Lifshitz equation [25], expressed as a torque equation involving the effective field and the magnetization itself. Since our aim is to find the energy density in a conservative system, we derive the equations of motion from the Hamilton equations.

2.2.1. HDMM for a Macrospin System. The equation of motion for the magnetic systems under investigation will be derived by following semiclassical approach. Also, the model will be first derived by considering the so-called macrospin approximation, where the material is thought as uniformly magnetized and represented by a single dipole momentum.

As known from classical mechanics, in the presence of fixed constraints and conservative sources, the system Hamiltonian $\mathscr{H}$ coincides with the total mechanical energy $\widetilde{E}$, namely, $\mathscr{H} \equiv \widetilde{E}=T-U$, where $T$ is the kinetic energy and $U$ is the potential expressed as the opposite of the potential energy $V$. By defining the Lagrangian variables of the problem with $q_{n}$, where $n=1,2, \ldots$ is the number of degrees of freedom corresponding to the dynamic variables, and the corresponding conjugate momenta with $p_{n}$, the Hamilton equations in the $2 n$ canonical variables $\left(q_{n}, p_{n}\right)$ take the form [15]

$$
\begin{gathered}
\frac{\partial q_{n}}{\partial t}=\frac{\partial \mathscr{H}}{\partial p_{n}}, \\
\frac{\partial p_{n}}{\partial t}=-\frac{\partial \mathscr{H}}{\partial q_{n}} .
\end{gathered}
$$

The direction of the magnetic dipole moment of thekth cell is given by (3). For the specific case, the dynamic variables 
referred to the $k$-cell are the small deviations from equilibrium of the azimuthal and polar angles given by

$$
q_{1}=\delta \phi, \quad q_{2}=\delta \theta,
$$

where 1 (2) labels the first (second) variable.

To determine the conjugate momenta, the expression of the angular momentum is needed. We recall the relation between the angular momentum $\vec{l}_{\Omega}$ and the magnetic momentum $\vec{\mu}$ by referring to a a rigid body with a fixed point $\Omega$ (see Figure 1), namely,

$$
\vec{l}_{\Omega}=\frac{1}{\gamma} \vec{\mu}=\frac{v_{c} M_{s}}{\gamma} \vec{m}
$$

where $\gamma$ is the gyromagnetic ratio and $v_{c}$ the volume of the magnetic moment to the case. Indeed, since $q_{1}$ represents a rotation about the $z$-axis of the magnetic dipole, its conjugate momentum $p_{1}$ corresponds to the $z$ component of the variation of the angular momentum, namely,

$$
p_{1}=\delta l_{z}=\frac{v_{c} M_{s}}{\gamma} \delta m_{z}=-\frac{v_{c} M_{s}}{\gamma} \sin \theta \delta \theta,
$$

where $\vec{m}=(\sin \theta \cos \phi, \sin \theta \sin \phi, \cos \theta)$ is the unit magnetization vector, normalized to the saturation magnetization $M_{s}$. Following an analogous argument, the other momentum $p_{2}$ can be determined. Indeed, $q_{2}$ is a rotation of the dipole moment about an axis of unitary vector $\hat{\phi}^{\prime}=-\sin \phi \hat{e}_{1}+$ $\cos \phi \hat{e}_{2}$ in the $x-y$ plane at an angle $\hat{\phi}^{\prime}=\phi+\pi / 2$ from the $x$-axis. Therefore, $p_{2}$ corresponds to the projection of the variation of the angular momentum along the $\hat{\phi}^{\prime}$ vector, namely,

$$
\begin{aligned}
p_{2} & =\delta \overrightarrow{l_{\Omega}} \cdot \widehat{\phi^{\prime}}=\frac{v_{c} M_{s}}{\gamma}\left(\delta \vec{m} \cdot \hat{\phi}^{\prime}\right) \\
& =-\frac{v_{c} M_{s}}{\gamma}\left(\frac{\partial \vec{m}}{\partial \phi} \delta \phi+\frac{\partial \vec{m}}{\partial \theta} \delta \theta\right) \cdot \hat{\phi}^{\prime} \\
& =\frac{v_{c} M_{s}}{\gamma} \sin \theta \delta \phi .
\end{aligned}
$$

By substituting (20), (22a), and (22b) into the first Hamilton equation (cf. (19)), the following system of equations is obtained:

$$
\begin{aligned}
\dot{\delta \phi} & =-\frac{\gamma}{v_{c} M_{s} \sin \theta} \mathscr{H}_{\delta \theta}, \\
\dot{\delta} \theta & =\frac{\gamma}{v_{c} M_{s} \sin \theta} \mathscr{H}_{\delta \phi},
\end{aligned}
$$

where the dot notation stands for the time derivative and, at the right-hand side, the first derivatives of the energy with respect to the mechanical variables appear. By introducing the energy density $E=\widetilde{E} / V$ (keeping in mind that $\mathscr{H}=\widetilde{E}$ ) and expanding it in a power Taylor expansion around the equilibrium up to the second order, it yields

$$
E=E_{0}+\frac{1}{2}\left[E_{\phi \phi}(\delta \phi)^{2}+2 E_{\phi \theta} \delta \phi \delta \theta+E_{\theta \theta}(\delta \theta)^{2}\right]
$$

where $E_{0}$ is the constant zero-order term that is inessential, the first-order terms vanish at equilibrium, and $E_{\alpha \beta}$ represent the second derivatives calculated at equilibrium $\left(E_{\alpha \beta}=\right.$ $\partial^{2} E / \partial \delta \alpha \partial \delta \beta$ with $\alpha, \beta=\phi, \theta$ ). By using (23) and (24), we obtain

$$
\begin{gathered}
\dot{\delta \phi}=-\frac{\gamma}{M_{s} \sin \theta}\left[E_{\theta \phi} \delta \phi+E_{\theta \theta} \delta \theta\right], \\
\dot{\delta \theta}=\frac{\gamma}{M_{s} \sin \theta}\left[E_{\phi \phi} \delta \phi+E_{\phi \theta} \delta \theta\right] .
\end{gathered}
$$

By inserting the time dependence in the form $e^{i \omega t}$, where $\omega$ is the angular frequency of the given collective mode, the system of equations of motion reads

$$
\begin{gathered}
-\frac{E_{\theta \phi}}{\sin \theta} \delta \phi-\frac{E_{\theta \theta}}{\sin \theta} \delta \theta-\tilde{\lambda} \delta \phi=0, \\
\frac{E_{\phi \phi}}{\sin \theta} \delta \phi+\frac{E_{\phi \theta}}{\sin \theta} \delta \theta-\tilde{\lambda} \delta \theta=0 .
\end{gathered}
$$

The linear and homogeneous system of equations expressed in (26) can be written as an eigenvalue problem

$$
\underline{\underline{C}} \vec{v}=\tilde{\lambda} \vec{v}
$$

with $\tilde{\lambda}=i\left(M_{s} / \gamma\right) \omega$ the complex eigenvalues of the problem,

$$
\underline{\underline{C}}=\left[\begin{array}{cc}
-\frac{E_{\theta \phi}}{\sin \theta} & -\frac{E_{\theta \theta}}{\sin \theta} \\
\frac{E_{\phi \phi}}{\sin \theta} & \frac{E_{\phi \theta}}{\sin \theta}
\end{array}\right],
$$

being $\underline{\underline{C}}$ a real, but not symmetric, matrix and $\vec{v}=(\delta \phi, \delta \theta)^{T}$.

However, the system of motion equations (26) can be also recast as a complex generalized Hermitian eigenvalue problem

$$
\underline{\underline{A} \vec{v}}=\lambda \underline{\underline{B}} \vec{v}
$$

where $\underline{B}$ is a Hessian matrix expressed by the second derivatives of the energy density at equilibrium. In particular

$$
\begin{gathered}
\underline{\underline{B}}=\left[\begin{array}{cc}
E_{\phi \phi} & E_{\phi \theta} \\
E_{\theta \phi} & E_{\theta \theta}
\end{array}\right], \\
\underline{\underline{A}}=\left[\begin{array}{cc}
0 & i \sin \theta \\
-i \sin \theta & 0
\end{array}\right] .
\end{gathered}
$$

It should be noticed that the matrix $\underline{\underline{A}}$ is Hermitian, whereas $\underline{\underline{B}}$ is real and symmetric, so that all the corresponding eigenvalues $\lambda=\gamma / M_{s} \omega$ are real quantities.

2.2.2. HDMM for an Isolated System Composed by N Interacting Magnetic Momenta. Equation (24) can be generalized to the case of $N$ interacting magnetic momenta (where each momentum is identified with a micromagnetic cell) taking the form

$$
E=E_{0}+\frac{1}{2} \sum_{n=1}^{N} \sum_{l=1}^{N}\left[E_{\phi_{n} \phi_{l}} \delta \phi_{n} \delta \phi_{l}+2 E_{\phi_{n} \theta_{l}} \delta \phi_{n} \delta \theta_{l}+E_{\theta_{n} \theta_{l}} \delta \theta_{n} \delta \theta_{l}\right] .
$$


In (31), the total energy density is given by $E=E_{\text {ext }}+E_{\text {exch }}+$ $E_{\mathrm{dmg}}+E_{\text {ani }}$ where the different contributions are expressed in (4), (6), (7), and (9), respectively. By substituting (31) into (23), we get

$$
\begin{gathered}
\delta \dot{\phi}_{k}=-\frac{\gamma}{M_{s} \sin \theta_{k}} \sum_{l=1}^{N}\left[E_{\theta_{k} \phi_{l}} \delta \phi_{l}+E_{\theta_{k} \theta_{l}} \delta \theta_{l}\right], \\
\delta \dot{\theta}_{k}=\frac{\gamma}{M_{s} \sin \theta_{k}} \sum_{l=1}^{N}\left[E_{\phi_{k} \phi_{l}} \delta \phi_{l}+E_{\phi_{k} \theta_{l}} \delta \theta_{l}\right] .
\end{gathered}
$$

By introducing the time dependence in the form $e^{i \omega t}$, the system of equations of motion is composed by the following $2 N$ linear and homogeneous equations for $k=1 \cdots N$ :

$$
\begin{gathered}
\sum_{l=1}^{N}\left(-\frac{E_{\theta_{k} \phi_{l}}}{\sin \theta_{k}}\right) \delta \phi_{l}+\sum_{l=1}^{N}\left(-\frac{E_{\theta_{k} \theta_{l}}}{\sin \theta_{k}}\right) \delta \theta_{l}-\tilde{\lambda} \delta \phi_{k}=0, \\
\sum_{l=1}^{N}\left(\frac{E_{\phi_{k} \phi_{l}}}{\sin \theta_{k}}\right) \delta \phi_{l}+\sum_{l=1}^{N}\left(\frac{E_{\phi_{k} \theta_{l}}}{\sin \theta_{k}}\right) \delta \theta_{l}-\tilde{\lambda} \delta \theta_{k}=0 .
\end{gathered}
$$

The unknown factors $\delta \phi_{l}, \delta \theta_{l}$ represent the eigenvectors of the problem and are expressed by the small angular deviation from the equilibrium position of the azimuthal $\left(\phi_{l}\right)$ and polar $\left(\theta_{l}\right)$ angles in the $l$ th micromagnetic cell. The system above has a solution only if the determinant is zero. By suitable exchanges of rows (columns), the linear and homogeneous system of equations expressed in (33) can be written as an eigenvalue problem in analogy with the case of a macrospin system

$$
\underline{\underline{C}} \vec{v}=\tilde{\lambda} \vec{v}
$$

In (34), $\vec{v}$ is the set of the unknown factors representing the eigenvectors of the problem that take the form

$$
\vec{v}=\left(\delta \phi_{1}, \delta \theta_{1}, \delta \phi_{2}, \delta \theta_{2}, \ldots, \delta \phi_{N}, \delta \theta_{N}\right)^{T}
$$

$\underline{\underline{C}}$ is the matrix whose elements are expressed as

$$
\left.\begin{array}{rl}
C_{2 k-1,2 l-1} & =-\frac{E_{\theta_{k} \phi_{l}}}{\sin \theta_{k}} \\
C_{2 k-1,2 l} & =-\frac{E_{\theta_{k} \theta_{l}}}{\sin \theta_{k}} \\
C_{2 k, 2 l-1} & =\frac{E_{\phi_{k} \phi_{l}}}{\sin \theta_{k}} \\
C_{2 k, 2 l} & =\frac{E_{\phi_{k} \theta_{l}}}{\sin \theta_{k}}
\end{array}\right\} k=1 \cdots N, l=1 \cdots N .
$$

Analogously to the case of a macrospin system, the matrix $\underline{C}$ is real but not symmetric, also in this case the eigenvalues (and not only the eigenvectors) are complex. This matrix can be indeed seen as composed by two submatrices $2 \times 2$ for each pair of values $(k, l)$. In the diagonal submatrices $(k=l)$ the following relation is verified:

$$
C_{2 k-1,2 k-1}=-C_{2 k, 2 k}
$$

For the elements of two different submatrices $(k, l)$ and $(l, k)$, that are not diagonal $(k \neq l)$, the following symmetries hold:

$$
\begin{gathered}
\sin \theta_{k} C_{2 k-1,2 l-1}=-\sin \theta_{l} C_{2 l, 2 k}, \\
\sin \theta_{k} C_{2 k-1,2 l}=\sin \theta_{l} C_{2 l-1,2 k}, \\
\sin \theta_{k} C_{2 k, 2 l-1}=\sin \theta_{l} C_{2 l, 2 k-1}, \\
\sin \theta_{k} C_{2 k, 2 l}=-\sin \theta_{l} C_{2 l-1,2 k-1} .
\end{gathered}
$$

As for the macrospin approximation, the equation of motion can be recast as a complex generalized Hermitian eigenvalue problem

$$
\underline{\underline{A}} \vec{v}=\lambda \underline{\underline{B}} \vec{v}
$$

where $\underline{\underline{B}}$ is a Hessian matrix expressed by the second derivatives of the energy density at equilibrium. $\underline{\underline{B}}$ is given by

$$
\left.\begin{array}{c}
B_{2 k-1,2 l-1}=E_{\phi_{k} \phi_{l}} \\
B_{2 k-1,2 l}=E_{\phi_{k} \theta_{l}} \\
B_{2 k, 2 l-1}=E_{\theta_{k} \phi_{l}} \\
B_{2 k, 2 l}=E_{\theta_{k} \theta_{l}}
\end{array}\right\} k=1 \cdots N, l=1 \cdots N
$$

where the matrix $\underline{B}$ is, again, real and symmetric. Moreover, since the static magnetization corresponds to a minimum of the energy and the matrix $\underline{B}$ is its Hessian, the matrix $\underline{B}$ is also positive defined. Instead, the matrix $\underline{\underline{A}}$ has the following form:

$$
\underline{\underline{A}}=\left[\begin{array}{ccccc}
0 & i \sin \theta_{1} & 0 & 0 & \cdots \\
-i \sin \theta_{1} & 0 & 0 & 0 & \cdots \\
0 & 0 & 0 & i \sin \theta_{2} & \cdots \\
0 & 0 & -i \sin \theta_{2} & 0 & \cdots \\
\cdots & & & & \cdots
\end{array}\right]
$$

The matrix $A$ is Hermitian. This allows us to solve the system as a complex generalized Hermitian eigenvalue problem which admits only real eigenvalues. To further reduce the computational time, it is possible to evaluate only some eigenvalues and eigenvectors that are in a specific range.

Once the eigenvectors $\vec{v}$ are obtained, the dynamic magnetization $\delta \vec{m}_{k}$ in the $k$ th micromagnetic cell expressed in Cartesian coordinates and in unit of $M_{S}$ is given by

$$
\begin{aligned}
\delta \vec{m}_{k}=( & -\sin \theta_{k} \sin \phi_{k} \delta \phi_{k}+\cos \theta_{k} \cos \phi_{k} \delta \theta_{k}, \\
& \left.\sin \theta_{k} \cos \phi_{k} \delta \phi_{k}+\cos \theta_{k} \sin \phi_{k} \delta \theta_{k},-\sin \theta_{k} \delta \theta_{k}\right)
\end{aligned}
$$

For each solution of the eigenvalue problem, the collection of all $\delta \vec{m}_{k}$ defines the mode profile. It must be remarked that $\delta \vec{m}_{k}$ is a complex vector, because $\delta \theta_{k}, \delta \phi_{k}$ are, in general, complex.

2.3. Equations of Motion for Interacting Magnetic Particles. Let us suppose to have a $2 \mathrm{D}$ periodic array of interacting nanodots characterized by the primitive vectors $\vec{a}_{1}$ and $\vec{a}_{2}$; 
for example, for the specific case of a rectangular lattice their values are

$$
\vec{a}_{1}=\lambda_{x} \hat{x}, \quad \vec{a}_{2}=\lambda_{y} \hat{y},
$$

where $\lambda_{x}$ and $\lambda_{y}$ represent the periodicity along $x$-axis and $y$-axis, respectively. The primitive vectors of the reciprocal lattice are $\vec{b}_{1}$ and $\vec{b}_{2}$

$$
\begin{aligned}
& \vec{b}_{1}=2 \pi \frac{\left(\vec{a}_{2} \times \vec{a}_{1}\right) \times \vec{a}_{2}}{\left(\vec{a}_{1} \times \vec{a}_{2}\right)^{2}}, \\
& \vec{b}_{2}=2 \pi \frac{\left(\vec{a}_{1} \times \vec{a}_{2}\right) \times \vec{a}_{2}}{\left(\vec{a}_{1} \times \vec{a}_{2}\right)^{2}} .
\end{aligned}
$$

For the special case of a rectangular lattice the vectors become

$$
\begin{aligned}
& \vec{b}_{1}=\frac{2 \pi}{\lambda_{x}} \hat{x}, \\
& \vec{b}_{2}=\frac{2 \pi}{\lambda_{y}} \hat{y} .
\end{aligned}
$$

Due to the analogy with the Bloch wave (analogy, not equality, because the wave function has not a physical meaning, contrarily to the magnetization), it is possible to write the following periodicity rule valid for the dynamic magnetization:

$$
\delta \vec{m}(\vec{r}+\vec{R})=e^{i \vec{K} \cdot \vec{R}} \delta \vec{m}(\vec{r}),
$$

where $\vec{R}$ is a vector of the particle lattice given by

$$
\vec{R}=i_{1} \vec{a}_{1}+i_{2} \vec{a}_{2} \quad i_{1}, i_{2} \in \mathbb{Z}, \quad i_{1}, i_{2}=-\frac{N_{i}}{2} \cdots \frac{N_{i}}{2}-1,
$$

and $\vec{r}$ can be confined into the first primitive cell centered in the first dot. The Bloch vector takes the following values:

$$
\vec{K}=\frac{n_{1}}{N_{1}} \vec{b}_{1}+\frac{n_{2}}{N_{2}} \vec{b}_{2}, \quad n_{i} \in \mathbb{Z}, \quad n_{i}=-\frac{N_{i}}{2} \cdots \frac{N_{i}}{2}-1 .
$$

$N_{1}, N_{2} \in \mathbb{N}$ indicate the number of primitive cells $n$ in direction $\vec{a}_{1}$ and $\vec{a}_{2}$, respectively. In this scheme, both $N_{1}$ and $N_{2}$ are taken as even numbers. In order to confirm the hypothesis on the dynamic magnetization $N_{1}, N_{2}$ must be very large.

If the magnetizations of different primitive cells and the different micromagnetic cells were independent, that is no periodicity rule were present, then one would have a dynamic system with variables $\theta_{k \vec{R}}$ and $\phi_{k \vec{R}}$, where the $k$ index changes inside the magnetic particle and $\vec{R}$ can assume the values indicated in (47). In this case, the linear and homogeneous system of $2 \mathrm{~N}$ equations of motion is

$$
\begin{gathered}
\sum_{l, \overrightarrow{R^{\prime}}}\left(-\frac{E_{\theta_{k \vec{R}} \phi_{l \vec{R}^{\prime}}}}{\sin \theta_{k \vec{R}}}\right) \delta \phi_{l \overrightarrow{R^{\prime}}}+\sum_{l, \overrightarrow{R^{\prime}}}\left(-\frac{E_{\theta_{k \vec{R}} \theta_{l \vec{R}^{\prime}}}}{\sin \theta_{j k \vec{R}}}\right) \delta \theta_{l \overrightarrow{R^{\prime}}}-\lambda \delta \phi_{k \vec{R}}=0, \\
\sum_{l, \overrightarrow{R^{\prime}}}\left(\frac{E_{\phi_{k \vec{R}} \phi_{l \vec{R}^{\prime}}}}{\sin \theta_{k \vec{R}}}\right) \delta \phi_{l \overrightarrow{R^{\prime}}}+\sum_{l, \overrightarrow{R^{\prime}}}\left(\frac{E_{\phi_{k \vec{R}} \theta_{l \vec{R}^{\prime}}}}{\sin \theta_{k \vec{R}}}\right) \delta \theta_{l \overrightarrow{R^{\prime}}}-\lambda \delta \theta_{k \vec{R}}=0,
\end{gathered}
$$

where $k=1 \cdots N$ and the sums over $l$ and $\vec{R}^{\prime}$ are on the same values. Instead, thanks to the Bloch condition expressed in (46), one can consider the equations only at $\vec{R}=0$; moreover, taking into account the same condition, the variables appearing for $\vec{R}^{\prime} \neq 0$ can be replaced by using the same condition. Now, when rewriting the system, the index $\vec{R}$ is omitted when it has value equal to 0 or it is irrelevant. Owing to these considerations, the system given in (49) can be rewritten in the form [16]

$$
\begin{aligned}
& \sum_{l=1}^{N}\left(-\frac{\sum_{\vec{R}^{\prime}} E_{\theta_{k} \phi_{l \vec{R}^{\prime}}} e^{i \vec{K} \cdot \vec{R}^{\prime}}}{\sin \theta_{k}}\right) \delta \phi_{l}+\sum_{l=1}^{N}\left(-\frac{\sum_{\vec{R}^{\prime}} E_{\theta_{k} \theta_{l \vec{R}^{\prime}}} e^{i \vec{K} \cdot \vec{R}^{\prime}}}{\sin \theta_{k}}\right) \delta \theta_{l} \\
& \quad-\tilde{\lambda} \delta \phi_{k}=0, \\
& \sum_{l=1}^{N}\left(\frac{\sum_{\vec{R}^{\prime}} E_{\phi_{k} \phi_{l \vec{R}^{\prime}}} e^{i \vec{K} \cdot \vec{R}^{\prime}}}{\sin \theta_{k}}\right) \delta \phi_{l}+\sum_{l=1}^{N}\left(\frac{\sum_{\vec{R}^{\prime}} E_{\phi_{k} \theta_{l \vec{R}^{\prime}}} e^{i \vec{K} \cdot \vec{R}^{\prime}}}{\sin \theta_{k}}\right) \delta \theta_{l} \\
& \quad-\tilde{\lambda} \delta \theta_{k}=0 .
\end{aligned}
$$

Equation (50) is similar to (33) by making the following replacement:

$$
E_{\alpha_{k} \beta_{l}} \longrightarrow \sum_{\vec{R}^{\prime}} e^{i \vec{K} \cdot \vec{R}^{\prime}} E_{\alpha_{k 0} \beta_{l \vec{R}^{\prime}}}
$$

and recalling that now the energy is referred to the whole system of particles. Like for the case of the isolated magnetic particle, also for the case of interacting magnetic particles the system of linear and homogeneous equations given in (50) can be written as an eigenvalue problem which in turn can be cast as a complex generalized Hermitian eigenvalue problem.

The symmetry for the matrix elements that was valid for a single primitive cell now is not respected except for $\vec{K}=0$ or $\vec{K}=\vec{G} / 2$ with $\vec{G}$ a translational reciprocal vector:

$$
\begin{aligned}
\sum_{\overrightarrow{R^{\prime}}} e^{i \vec{K} \cdot \vec{R}^{\prime}} E_{\alpha_{k \overrightarrow{0}} \beta_{l \vec{R}^{\prime}}} & =\sum_{\vec{R}^{\prime}} e^{i \vec{K} \cdot \vec{R}^{\prime}} E_{\beta_{l \vec{R}^{\prime}} \alpha_{k \overrightarrow{0}}}=\sum_{\overrightarrow{R^{\prime}}} e^{i \vec{K} \cdot \overrightarrow{R^{\prime}}} E_{\beta_{l \overrightarrow{0}} \alpha_{k-\vec{R}^{\prime}}} \\
& =\sum_{\vec{R}^{\prime}} e^{-i \vec{K} \cdot \vec{R}^{\prime \prime}} E_{\beta_{l \overrightarrow{0}} \alpha_{k \vec{R}^{\prime \prime}}} \\
& \neq \sum_{\overrightarrow{R^{\prime}}} e^{i \vec{K} \cdot \vec{R}^{\prime}} E_{\beta_{l \overrightarrow{0}} \alpha_{k \vec{R}^{\prime}}}, \quad \vec{K} \neq \overrightarrow{0}, \vec{K} \neq \frac{\vec{G}}{2},
\end{aligned}
$$

with $\vec{R}^{\prime \prime}=-\vec{R}^{\prime}$. The primitive cell has at the centre a single dot that occupies only a part of it. The interdot exchange coupling is zero. Thanks to the last consideration and to the fact that derivatives of Zeeman, exchange, anisotropy, energy density are referred only to the cell of the first variable $\left(\alpha_{k \overrightarrow{0}}\right)$ with $\alpha_{k \vec{R}}=\theta_{k \vec{R}}, \phi_{k \vec{R}}$ or at most to the nearest neighbour, all terms of the sum in (51) with $\vec{R}^{\prime} \neq 0$ are zero. Hence, for these energy density terms, the equations are the same as those of the single particle case and the same occurs for their corresponding derivatives appearing in the equations of motion. The only energy density term that differs from the 
one obtained for the isolated element is the demagnetizing energy density. For a system of interacting nanoparticles, the demagnetizing energy density can be written as

$$
E_{\mathrm{dmg}}=\frac{1}{2} \mu_{0} \sum_{\vec{R}, \vec{R}^{\prime}, k, k^{\prime}} \vec{m}_{k}(\vec{R}) \underline{\underline{N}}\left(\vec{R}, \vec{R}^{\prime}, k, k^{\prime}\right) \vec{m}_{k^{\prime}}\left(\vec{R}^{\prime}\right) .
$$

Due to its rather complex expression, it is useful to give the derivation also of the first derivative of $E_{\mathrm{dmg}}$ like for the case of the isolated nanoelement.

In order to calculate the first derivative, the properties of the demagnetizing tensor must be considered

$$
\begin{aligned}
\langle v|N| \omega\rangle & =\left\langle\omega\left|N^{+}\right| v\right\rangle^{*}=\left\langle\omega\left|N^{+}\right| v\right\rangle \\
& =\left\langle\omega\left|N^{t}\right| v\right\rangle=\langle\omega|N| v\rangle,
\end{aligned}
$$

since $N$ is real and symmetric and

$$
\begin{aligned}
\underline{\underline{N}}\left(\vec{R}, \vec{R}^{\prime}, k, k^{\prime}\right) & =\underline{\underline{N}}\left(\vec{R}^{\prime}-\vec{R}+\vec{r}_{k^{\prime}}-\vec{r}_{k}\right) \\
& =\underline{\underline{N}}\left(-\vec{R}^{\prime}+\vec{R}-\vec{r}_{k^{\prime}}+\vec{r}_{k}\right)=\underline{\underline{N}}\left(\vec{R}^{\prime}, \vec{R}, k^{\prime}, k\right),
\end{aligned}
$$

thanks to the inversion symmetry. Hence, the first derivative of the energy is

$$
\begin{aligned}
& \frac{\partial E_{\mathrm{dmg}}}{\partial \delta \alpha_{k}(\overrightarrow{0})} \\
& =\mu_{0} \frac{M_{s}^{2}}{2}\left[\sum_{\substack{\vec{R}^{\prime} k^{\prime} \\
\left(\vec{R}^{\prime}, k^{\prime}\right) \neq(\overrightarrow{0}, k)}} \vec{m}_{k^{\prime}}\left(\vec{R}^{\prime}\right) \cdot \underline{\underline{N}}\left(\vec{R}^{\prime}, \overrightarrow{0}, k^{\prime}, k\right) \frac{\partial \vec{m}_{k}(\overrightarrow{0})}{\partial \delta \alpha_{k}(\overrightarrow{0})}\right. \\
& +\sum_{\substack{\vec{R}^{\prime} k^{\prime} \\
\left(\vec{R}^{\prime}, k^{\prime}\right) \neq(\hat{0}, k)}} \frac{\partial \vec{m}_{k}(\overrightarrow{0})}{\partial \delta \alpha_{k}(\overrightarrow{0})} \cdot \underline{\underline{N}}\left(\overrightarrow{0}, \vec{R}^{\prime}, k^{\prime}, k\right) \vec{m}_{k^{\prime}}\left(\vec{R}^{\prime}\right) \\
& \left.+\frac{\partial}{\partial \delta \alpha_{k}(\overrightarrow{0})}\left(\vec{m}_{k}(\overrightarrow{0}) \cdot \underline{\underline{N}}(\overrightarrow{0}, \overrightarrow{0}, k, k) \vec{m}_{k}(\overrightarrow{0})\right)\right] \\
& =\mu_{0} M_{s}^{2}\left[\sum_{\substack{\vec{R}^{\prime} k^{\prime} \\
\left(\vec{R}^{\prime}, k^{\prime}\right) \neq(\overrightarrow{0}, k)}} \vec{m}_{k^{\prime}}\left(\vec{R}^{\prime}\right) \cdot \underline{\underline{N}}\left(\overrightarrow{R^{\prime}}, \overrightarrow{0}, k^{\prime}, k\right) \frac{\partial \vec{m}_{k}(\overrightarrow{0})}{\partial \delta \alpha_{k}(\overrightarrow{0})}\right. \\
& \left.+\vec{m}_{k}(\overrightarrow{0}) \cdot \underline{\underline{N}}(\overrightarrow{0}, \overrightarrow{0}, k, k) \frac{\partial \vec{m}_{k}(\overrightarrow{0})}{\partial \delta \alpha_{k}(\overrightarrow{0})}\right] .
\end{aligned}
$$

The second derivative takes the form

$$
\begin{aligned}
& \frac{\partial^{2} E_{\mathrm{dmg}}}{\partial \delta \alpha_{k}(\overrightarrow{0}) \partial \delta \beta_{k}(\vec{R})}
\end{aligned}
$$

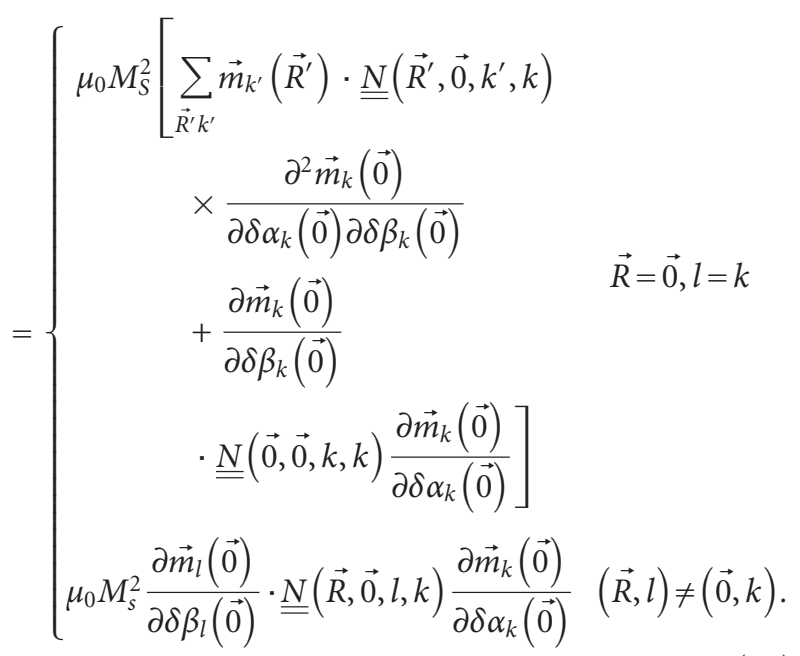

In the sum it is included the case in which $\left(\vec{R}^{\prime}, k^{\prime}\right)=(\overrightarrow{0}, k)$ that is generated from the derivative of the second term in the expression of the first derivative given above. Now it is possible to calculate the terms that enter into the system of (50), starting with the one corresponding to $l=k$

$$
\begin{aligned}
& \sum_{\vec{R}} e^{i \vec{K} \cdot \vec{R}} E_{\alpha_{k \overrightarrow{0}} \beta_{l \vec{R}}}=\sum_{\vec{R}} e^{i \vec{K} \cdot \vec{R}} E_{\alpha_{k} \overrightarrow{0} \beta_{k \vec{R}}} \\
& =\mu_{0} M_{s}^{2}\left[\sum_{\vec{R}^{\prime} k^{\prime}} \vec{m}_{k^{\prime}}(\overrightarrow{0}) \cdot \underline{\underline{N}}\left(\overrightarrow{R^{\prime}}, \overrightarrow{0}, k^{\prime}, k\right)\right. \\
& \times \frac{\partial^{2} \vec{m}_{k}(\overrightarrow{0})}{\partial \delta \alpha_{k}(\overrightarrow{0}) \partial \delta \beta_{k}(\overrightarrow{0})}+\frac{\partial \vec{m}_{k}(\overrightarrow{0})}{\partial \delta \beta_{k}(\overrightarrow{0})} \\
& \left.\cdot \underline{\underline{N}}(\overrightarrow{0}, \overrightarrow{0}, k, k) \frac{\partial \vec{m}_{k}(\overrightarrow{0})}{\partial \delta \alpha_{k}(\overrightarrow{0})}\right] \\
& +\mu_{0} M_{s}^{2} \sum_{\vec{R} \neq \overrightarrow{0}} e^{i \vec{K} \cdot \vec{R}} \frac{\partial \vec{m}_{k}(\overrightarrow{0})}{\partial \delta \beta_{k}(\overrightarrow{0})} \\
& \cdot \underline{\underline{N}}(\vec{R}, \overrightarrow{0}, k, k) \frac{\partial \vec{m}_{k}(\overrightarrow{0})}{\partial \delta \alpha_{k}(\overrightarrow{0})} \\
& =\mu_{0} M_{s}^{2} \sum_{\vec{R}}\left[\sum_{k^{\prime}} \vec{m}_{k^{\prime}}(\overrightarrow{0}) \cdot \underline{\underline{N}}\left(\vec{R}, \overrightarrow{0}, k^{\prime}, k\right)\right. \\
& \times \frac{\partial^{2} \vec{m}_{k}(\overrightarrow{0})}{\partial \delta \alpha_{k}(\overrightarrow{0}) \partial \delta \beta_{k}(\overrightarrow{0})}
\end{aligned}
$$




$$
\begin{aligned}
& +e^{i \vec{K} \cdot \vec{R}} \frac{\partial \vec{m}_{k}(\overrightarrow{0})}{\partial \delta \beta_{k}(\overrightarrow{0})} \\
& \left.\cdot \underline{\underline{N}}(\vec{R}, \overrightarrow{0}, k, k) \frac{\partial \vec{m}_{k}(\overrightarrow{0})}{\partial \delta \alpha_{k}(\overrightarrow{0})}\right] .
\end{aligned}
$$

Equation (57a) is obtained by taking into account (46), by including the term corresponding to $\vec{R}=0$ into the sum performed over $\vec{R} \neq 0$ and by considering that both the static magnetization and the dynamic magnetization do not depend on $\vec{R}$. Indeed, second derivatives are calculated at equilibrium and the exponential $e^{i \vec{K} \cdot \vec{R}}$ appears on both the numerator and the denominator of the derivative.

When $l \neq k$, the term turns out to be

$$
\begin{aligned}
& \sum_{\vec{R}} e^{i \vec{K} \cdot \vec{R}} E_{\alpha_{k 0} \beta_{l \vec{R}}} \\
& \quad=\mu_{0} M_{s}^{2}\left[\sum_{\vec{R}} e^{i \vec{K} \cdot \vec{R}} \frac{\partial \vec{m}_{l}(\overrightarrow{0})}{\partial \delta \beta_{l}(\overrightarrow{0})} \cdot \underline{\underline{N}}(\vec{R}, \overrightarrow{0}, l, k) \frac{\partial \vec{m}_{k}(\overrightarrow{0})}{\partial \delta \alpha_{k}(\overrightarrow{0})}\right] .
\end{aligned}
$$

Due to the properties of the demagnetizing tensor, the symmetry

$$
\sum_{\overrightarrow{R^{\prime}}} e^{i \vec{K} \cdot \vec{R}^{\prime}} E_{\alpha_{k \hat{0}} \beta_{\vec{l} \vec{R}^{\prime}}}=\sum_{\overrightarrow{R^{\prime}}} e^{i \vec{K} \cdot \vec{R}^{\prime}} E_{\alpha_{l \overrightarrow{0}} \beta_{k \vec{R}^{\prime}}},
$$

is fulfilled when $l=k$, but it is not fulfilled when $l \neq k$.

The formalism previously developed for interacting particles can be extended to a system of 2D antidots (ADs). In this case, it is necessary to add the exchange interaction between primitive cells [21]. In extended magnetic system like $\mathrm{AD}$ arrays, in addition to the usual nearest-neighbours exchange interaction between micromagnetic cells, the exchange contribution across the nearest-neighbours micromagnetic cells belonging to adjacent surface primitive cells must be taken into account. Hence, we recall the exchange energy density of (6)

$$
E_{\text {exch }}=A \sum_{k} \sum_{n} \frac{\left(1-\vec{m}_{k} \cdot \vec{m}_{n}\right)}{a_{k n}^{2}} .
$$

Here, the first sum runs over all the micromagnetic cells of the primitive cell and the second sum runs over the nearest neighbours of the $k$ th micromagnetic cell. When the $k$ th micromagnetic cell is on one of the edges (vertices) of the given primitive cell, the interaction with one (two) micromagnetic cell(s) belonging to the correct nearest primitive cell must be added.

\section{LDMM}

This section is devoted to the review of the LDMM approach through which we derive the generalized Lagrange equation

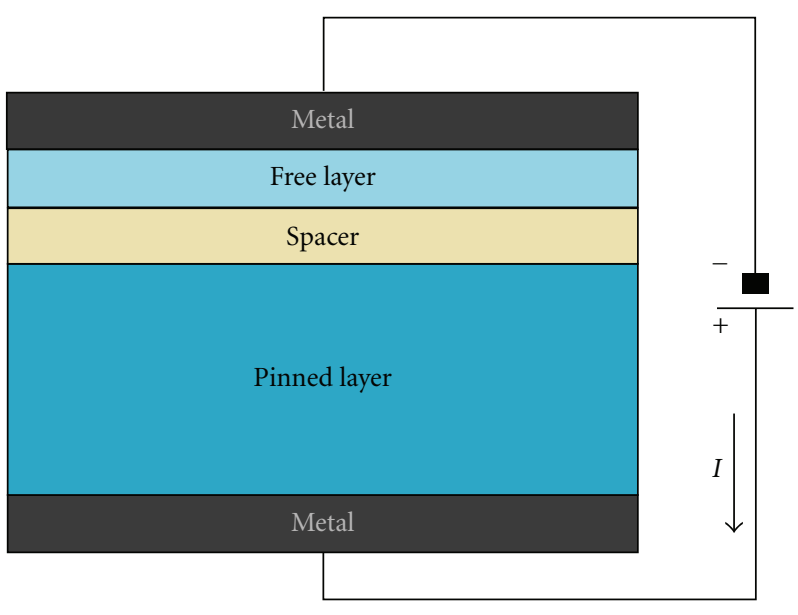

FIGURE 2: A schematic of a spin-valve nanopillar device.

in the presence of two dissipative effects arising from the "positive" intrinsic damping and the "negative" one induced by the current-driven spin-transfer torque [22]. As for the Hamiltonian approach, we limit our study to the dynamics taking place in the linear and autonomous regime.

3.1. Description of the Magnetic System and Equation of Motion. The magnetic systems in which such competing phenomena take place are generally referred to as spin-valve nanopillars. These are heterostructures composed by two ferromagnetic layers, having generally different thicknesses, separated by a nonmagnetic (metallic or insulating) spacer, which is used to decouple the exchange interactions between them. The thinner magnetic layer is generally referred to as "Free Layer" (FL), whereas the thicker one is called "Fixed Layer" or "Pinned Layer" (PL). By means of an external voltage source and metallic contacts applied at the top and bottom of the structure, a current flow traverses the structure along the normal-to-plane direction (see Figure 2).

In this kind of device, conservative effects arise from the previously mentioned classical micromagnetic contributions (exchange, demagnetizing, magnetocrystalline anisotropy, and Zeeman fields) together with the Ampere (or Oersted) field due to the current flow. This latter contribution, however, will be neglected for simplicity. In fact, the main goal of this section is to describe why and how a Lagrangian approach needs to be take into account when nonconservative forces act in the system. The inclusion of more sophisticated effects, such as the Ampere field, will become relevant when this approach will be further generalized to describe the dynamics occurring in the nonlinear regime of spin-wave generation.

Let us now briefly recall the governing equation of motion. When no dissipative contributions are taken into account, a persistent precessional motion of the magnetization vector $\vec{M}$ takes place. It is described by classical LandauLifshitz equation $\dot{\vec{M}}=\gamma\left(\vec{H}_{\text {eff }} \times \vec{M}\right)$, where $\vec{H}_{\text {eff }}$ is the effective field which accounts for all the above-mentioned contributions. 
On the other hand, nonconservative contributions arise from the material intrinsic dissipation and the spin-transfer torque induced by the current flow [26]. The former accounts for the phenomenologically introduced intrinsic Gilbert dissipation [27], the phenomenon by which the precessional motion of $\vec{M}$, excited by a given stimulus, relaxes towards its equilibrium state. The relaxation rate is proportional to a scalar quantity, called Gilbert constant $\alpha$. The torque exerted on the magnetization is generally represented by $\vec{T}_{\mathrm{ID}}=\left(\alpha / M_{S}\right)(\vec{M} \times \dot{\vec{M}})$.

Concerning the dissipative effects induced by the current flow, it has been extensively shown that this bias current can become spin-polarized in the direction of the magnetization vector of the thicker magnetic layer and can then transfer this induced spin angular momentum to the magnetization of the thinner magnetic layer. For a proper direction of the bias current $I$, this spin-transfer mechanism creates a torque which opposes to that induced by the Gilbert damping, creating an effective negative damping. The corresponding spin-transfer torque, derived by Slonczewski [28], can be expressed as $\vec{T}_{\mathrm{ST}}=\left(\sigma I / M_{S}\right)[\vec{M} \times(\vec{M} \times \vec{p})]$, where the unit vector $\vec{p}$ defines the direction of the spin polarization (in turn defined by the magnetization vector of the PL) and the constant $\sigma$ modulates the strength of the spin-torque effect. It is equal to $\sigma=\varepsilon g_{0} \mu_{B} / 2 e M_{S} S d$, where $\varepsilon$ is the spintorque efficiency (defined in [29]), $g_{0}$ is the Landè factor, $\mu_{B}$ is the Bohr magneton, $e$ is the absolute value of the electronic charge, $d$ is the thickness of the magnetic layer, and $S$ is the current-carrying area [30].

It should be mentioned that, because of the larger value of both saturation magnetization and thickness, the PL is not substantially affected by any current-driven magnetization dynamics, so that it is generally treated as it were fixed (or pinned) along its equilibrium direction. On the contrary, the FL's properties allow it to describe more easily several kinds of dynamics (e.g., switching [31], precession [32], domainwall motion [33], gyrotropic motion of vortex state [34]), as it were "free" to move.

Under these circumstances, the magnetization dynamics of the FL is governed by the Landau-Lifshitz-Gilbert-Slonczewski (LLGS) equation

$$
\dot{\vec{M}}=\gamma\left(\vec{H}_{\text {eff }} \times \vec{M}\right)+\frac{\alpha}{M_{S}}(\vec{M} \times \dot{\vec{M}})+\frac{\sigma I}{M_{S}}[\vec{M} \times(\vec{M} \times \vec{p})] .
$$

It has to be remarked that the equality (with opposite sign) of the two torques $\left(\vec{T}_{\mathrm{ID}}=-\vec{T}_{\mathrm{ST}}\right)$, achievable by means of a proper intensity and sign of current (the positive one, $I$ $>0$, which corresponds to a current flow moving from the PL to the FL), which in turn implies the fully compensation of the two dissipation mechanisms, yields the system in an out-of-equilibrium zero-dissipation stationary state (a limit cycle, using the notation of dynamic systems) (see Figure 3). In such a regime, the excitation of microwave spin waves becomes physically conceivable.

To derive the mathematical formulation of the LDMM, for the sake of simplicity, we write, first, the generalized Lagrange equation for the case of an isolated magnetic
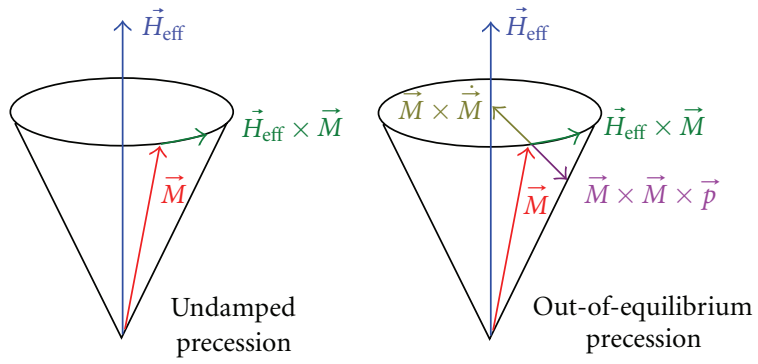

FIGURE 3: Schematic representation of undamped (in the absence of both Gilbert and current-induced damping) and out-ofequilibrium (in the presence of both Gilbert and current-induced damping) precessions. In this latter case, a limit cycle is described as well since the torques due to the intrinsic dissipation and the spintransfer-induced one (for a proper intensity and direction of the current) balance each other.

particle within the macrospin approximation. After that, we will generalize this approach for the case of an isolated particle composed by $N$ interacting momenta.

3.1.1. LDMM for a Macrospin System. We will preliminarily assume that the dynamics of the magnetization vector could be described through three degress of freedom $(\theta, \phi, \psi)$, as shown in Figure 1. In such a framework the generalized Lagrange equations read

$$
\begin{aligned}
& \frac{d}{d t}\left(\frac{\partial L}{\partial \dot{\phi}}\right)-\frac{\partial L}{\partial \phi}+\frac{\partial \mathfrak{I}_{\mathrm{ST}}}{\partial \dot{\phi}}+\frac{\partial \mathfrak{I}_{\mathrm{ID}}}{\partial \dot{\phi}}=0, \\
& \frac{d}{d t}\left(\frac{\partial L}{\partial \dot{\theta}}\right)-\frac{\partial L}{\partial \theta}+\frac{\partial \mathfrak{J}_{S \mathrm{~T}}}{\partial \dot{\theta}}+\frac{\partial \mathfrak{J}_{\mathrm{ID}}}{\partial \dot{\theta}}=0, \\
& \frac{d}{d t}\left(\frac{\partial L}{\partial \dot{\psi}}\right)-\frac{\partial L}{\partial \psi}+\frac{\partial \mathfrak{I}_{\mathrm{ST}}}{\partial \dot{\psi}}+\frac{\partial \mathfrak{I}_{\mathrm{ID}}}{\partial \dot{\psi}}=0
\end{aligned}
$$

where $L=T+U$ represents the Lagrangian of the system given by the sum of the kinetic energy $T$ and the potential $U$, whereas $\mathfrak{I}_{S T}$ and $\mathfrak{I}_{\text {ID }}$ are the dissipation functions related to the spin torque and the intrinsic damping, respectively.

Taking advantage of the explicit formulations of the energy contributions given in Section 2.1, we can rewrite the previous system by accounting for the relationship among the potential, the kinetic energy, and the conservative part $\widetilde{E}=T-U$ of the total mechanical energy (which in the conservative limit coincides with the Hamiltonian of the system $\mathcal{H}$ in the presence of fixed constraints). By substituting $L$ with $2 T-\widetilde{E}$, we thus obtain

$$
\begin{aligned}
& 2 \frac{d}{d t}\left(\frac{\partial T}{\partial \dot{\phi}}\right)-\frac{d}{d t}\left(\frac{\partial \widetilde{E}}{\partial \dot{\phi}}\right)-2 \frac{\partial T}{\partial \phi}+\frac{\partial \widetilde{E}}{\partial \phi}+\frac{\partial \mathfrak{I}_{\mathrm{ST}}}{\partial \dot{\phi}}+\frac{\partial \mathfrak{I}_{\mathrm{ID}}}{\partial \dot{\phi}}=0, \\
& 2 \frac{d}{d t}\left(\frac{\partial T}{\partial \dot{\theta}}\right)-\frac{d}{d t}\left(\frac{\partial \widetilde{E}}{\partial \dot{\theta}}\right)-2 \frac{\partial T}{\partial \theta}+\frac{\partial \widetilde{E}}{\partial \theta}+\frac{\partial \mathfrak{J}_{\mathrm{ST}}}{\partial \dot{\theta}}+\frac{\partial \mathfrak{I}_{\mathrm{ID}}}{\partial \dot{\theta}}=0, \\
& 2 \frac{d}{d t}\left(\frac{\partial T}{\partial \dot{\psi}}\right)-\frac{d}{d t}\left(\frac{\partial \widetilde{E}}{\partial \dot{\psi}}\right)-2 \frac{\partial T}{\partial \psi}+\frac{\partial \widetilde{E}}{\partial \psi}+\frac{\partial \mathfrak{J}_{\mathrm{ST}}}{\partial \dot{\psi}}+\frac{\partial \mathfrak{I}_{\mathrm{ID}}}{\partial \dot{\psi}}=0 .
\end{aligned}
$$


To solve the proposed problem, we need thus to explicitly find the expressions of the variables $T, \widetilde{E}, \mathfrak{I}_{\mathrm{ID}}$ and $\mathfrak{I}_{\mathrm{ST}}$, shown in (62), in Lagrangian coordinates.

The kinetic energy $T$ associated to the precessional motion of the magnetization vector can be computed by referring to the case of a rigid body with a fixed point $\Omega$ (see Figure 1). In this case, the rotational kinetic energy density is expressed as

$$
T=\frac{1}{2} \overrightarrow{l_{\Omega}} \cdot \vec{\omega}
$$

where $\vec{l}_{\Omega}$ is the angular momentum and $\vec{\omega}$ is the angular velocity vector. Notice that in the following the kinetic energy $T$, the dissipation functions $\mathfrak{I}_{\mathrm{ID}}$ and $\mathfrak{J}_{\mathrm{ST}}$, the work $d w$, and the energy losses $d w / d t$ are referred to their corresponding quantities per unit volume. In addition, we recall the definition of the energy density $E=\widetilde{E} / V$ given in Section 2.1. By virtue of the relationship between angular momentum $\vec{l}_{\Omega}$ and magnetic dipole momentum $\vec{\mu}\left(\vec{l}_{\Omega}=\right.$ $(1 / \gamma) \vec{\mu})$, and considering that this latter is directed along $\hat{e}_{3}^{\prime}$ (see Figure 1), the previous scalar product involves, in turn, the only component of $\vec{l}_{\Omega}$ along $\hat{e}_{3}$. It leads to

$$
T=\frac{1}{2} \frac{M_{S}}{\gamma}(\dot{\phi} \cos \theta+\dot{\psi}) \text {. }
$$

Equation (64) therefore represents the rotational kinetic energy $T=T(\theta, \phi, \psi)$ expressed in Lagrangian coordinates with $M_{S}=\mu / V$. The conservative part $E$ of the energy density accounts for all the standard micromagnetic contributions discussed previously. As shown in Section 2.1, all the contributions appearing in $E$ only depend on the Lagrangian variables $\theta, \phi$, but not on their derivatives, namely, $E=$ $E(\theta, \phi)$.

To derive the Lagrangian formulation of the dissipative contributions, let us start from the classical definition of the work $d w$ carried out by a magnetic system subject to a nonconservative force. As it is known, such a force has to be derived from the gradient of a dissipation function $\mathfrak{I}$ and the rate of energy losses associated to a dissipative torque can be thus expressed as

$$
\frac{d w}{d t}= \pm \dot{\vec{M}} \cdot \frac{\partial \mathfrak{I}}{\partial \dot{\vec{M}}}
$$

where the plus (minus) sign accounts for torques which act as "drain" ("source") of energy and refers to the case $\mathfrak{I}=\mathfrak{I}_{\text {ID }}$ $\left(\mathfrak{I}=\mathfrak{I}_{\mathrm{ST}}\right)$.

So, by multiplying the LLGS equation (60) by $\vec{H}_{\text {eff }}$ and assuming that the energy losses rates are small compared to the conservative (precessional) part, namely, $\dot{\vec{M}} \simeq-\gamma \mu_{0}(\vec{M} \times$ $\vec{H}_{\text {eff }}$ ), we deduce the following expressions for the dissipative function densities

$$
\begin{gathered}
\mathfrak{I}_{\mathrm{ID}}=\frac{\alpha}{2 \gamma M_{0}} \dot{\vec{M}}^{2}, \\
\mathfrak{I}_{\mathrm{ST}}=\frac{\sigma J}{\gamma M_{0}} \vec{p} \cdot(\vec{M} \times \dot{\vec{M}}) .
\end{gathered}
$$

It should be noticed that (66), which appears in the usual form of a Rayleigh-like dissipation function, is a positivedefinite form, as expected for a power dissipated through a viscous friction mechanism, whatever the magnetization configuration ( $\alpha, \gamma$, and $M_{s}$ are positive constants). On the other hand, the spin-torque dissipation function of (67) is a non-Rayleigh one and strongly depends, apart from the direction of the current flow $J$, on the relative magnetization configuration of the ferromagnetic layers $(\vec{M}$ and $\vec{p})$.

Finally, taking into account the expression of the timeindependent unit vector $\vec{p}=\left(\sin \Theta_{\mathrm{PL}} \cos \Phi_{\mathrm{PL}}, \sin \Theta_{\mathrm{PL}}\right.$ $\left.\sin \Phi_{\mathrm{PL}}, \cos \Theta_{\mathrm{PL}}\right)$, the explicit expressions of the dissipative functions densities are

$$
\begin{gathered}
\mathfrak{I}_{\mathrm{ID}}=\frac{\alpha M_{s}}{2 \gamma}\left(\dot{\theta}^{2}+\dot{\phi}^{2} \sin ^{2} \theta\right)=\mathfrak{I}_{\mathrm{ID}}(\theta, \phi), \\
\mathfrak{J}_{\mathrm{ST}}=\frac{\sigma J M_{s}}{\gamma}\left[f_{1}(\phi) \dot{\theta}+f_{2}(\theta, \phi) \dot{\phi}\right]=\mathfrak{I}_{\mathrm{ST}}(\theta, \phi),
\end{gathered}
$$

where

$$
\begin{gathered}
f_{1}(\phi)=\sin \Theta_{\mathrm{PL}} \sin \left(\Phi_{\mathrm{PL}}-\phi\right), \\
f_{2}(\theta, \phi)=\sin ^{2} \theta \cos \Theta_{\mathrm{PL}}-\cos \theta \sin \theta \sin \Theta_{\mathrm{PL}} \cos \left(\Phi_{\mathrm{PL}}-\phi\right) .
\end{gathered}
$$

We proceed by evaluating first the third Lagrange equation

$$
2 \frac{d}{d t}\left(\frac{\partial T}{\partial \dot{\psi}}\right)-\frac{d}{d t}\left(\frac{\partial E}{\partial \dot{\psi}}\right)-2 \frac{\partial T}{\partial \psi}+\frac{\partial E}{\partial \psi}+\frac{\partial \mathfrak{I}_{\mathrm{ST}}}{\partial \dot{\psi}}+\frac{\partial \mathfrak{I}_{\mathrm{ID}}}{\partial \dot{\psi}}=0 .
$$

In (71), we notice that the kinetic energy is the only term dependent on the intrinsic rotation, and, in particular, on its velocity $\dot{\psi}$, so that the previous equation reduces to

$$
2 \frac{d}{d t}\left(\frac{\partial T}{\partial \dot{\psi}}\right)=0
$$

which stands for a first integral of motion representing the conservation of the (only component of the) angular momentum

$$
2 \frac{\partial T}{\partial \dot{\psi}}=\frac{M_{S}}{\gamma}=\text { constant } \equiv \vec{l}_{\Omega} \cdot \vec{e}_{3} .
$$

Such a first integral also points out that our system can be described through only two degrees of freedom, as expected for the characterization of the dynamics of a vector having constant modulus which undergoes a precession (with a fixed point) onto a sphere. Taking into account this result, in the following we will use the parameters $\theta, \phi$.

The first two Lagrange equations read

$$
\begin{aligned}
& 2 \frac{d}{d t}\left(\frac{\partial T}{\partial \dot{\phi}}\right)-\frac{d}{d t}\left(\frac{\partial E}{\partial \dot{\phi}}\right)-2 \frac{\partial T}{\partial \phi}+\frac{\partial E}{\partial \phi}+\frac{\partial \mathfrak{I}_{\mathrm{ST}}}{\partial \dot{\phi}}+\frac{\partial \mathfrak{I}_{\mathrm{ID}}}{\partial \dot{\phi}}=0, \\
& 2 \frac{d}{d t}\left(\frac{\partial T}{\partial \dot{\theta}}\right)-\frac{d}{d t}\left(\frac{\partial E}{\partial \dot{\theta}}\right)-2 \frac{\partial T}{\partial \theta}+\frac{\partial E}{\partial \theta}+\frac{\partial \mathfrak{I}_{\mathrm{ST}}}{\partial \dot{\theta}}+\frac{\partial \mathfrak{I}_{\mathrm{ID}}}{\partial \dot{\theta}}=0
\end{aligned}
$$


which, substituting the corresponding expressions (the conservative part $E$ of the total energy density will be discussed later on in the text), become

$$
\begin{gathered}
-\frac{M_{s}}{\gamma} \sin \theta \dot{\theta}+\frac{\partial E}{\partial \phi}+\frac{\sigma J M_{s}}{\gamma} f_{2}+\frac{\alpha M_{s}}{\gamma} \dot{\phi} \sin ^{2} \theta=0, \\
\frac{M_{s}}{\gamma} \dot{\phi} \sin \theta+\frac{\partial E}{\partial \theta}+\frac{\sigma J M_{s}}{\gamma} f_{1}+\frac{\alpha M_{s}}{\gamma} \dot{\theta}=0 .
\end{gathered}
$$

Since in the present LDMM approach we are interested in the characterization of the linear dynamics of these magnetic current-driven auto-oscillatory systems, we adopt the classical formalism of small oscillations. By following this procedure, we linearize the system of (75) by considering small perturbations $(\delta \theta, \delta \phi)$ around the equilibrium configuration of the FL $(\Theta, \Phi)$, as done in (2), and expand the energy terms in Taylor series up to the second perturbative order.

By means of such a formalism, the system given in (75) leads to the set of generalized Lagrange equations

$$
\begin{aligned}
& -\frac{M_{s}}{\gamma} \sin \Theta \dot{\delta} \theta+\frac{\partial E}{\partial \delta \phi}+\frac{\sigma J M_{s}}{\gamma} \\
& \times[\chi(\Theta, \Phi) \delta \theta+\eta(\Theta, \Phi) \delta \phi+\nu(\Theta, \Phi)] \\
& \quad+\frac{\alpha M_{s}}{\gamma} \dot{\delta} \phi \sin ^{2} \Theta=0 \\
& \frac{M_{s}}{\gamma} \dot{\delta} \phi \sin \Theta+\frac{\partial E}{\partial \delta \theta}+\frac{\sigma J M_{s}}{\gamma}[\zeta(\Theta, \Phi) \delta \phi+\beta(\Theta, \Phi)] \\
& +\frac{\alpha M_{s}}{\gamma} \dot{\delta} \theta=0
\end{aligned}
$$

where

$$
\begin{aligned}
& \chi(\Theta, \Phi)=\sin 2 \Theta \cos \Theta_{\mathrm{PL}}-\cos 2 \Theta \sin \Theta_{\mathrm{PL}} \cos \left(\Phi_{\mathrm{PL}}-\Phi\right), \\
& \eta(\Theta, \Phi)=\cos \Theta \sin \Theta \sin \Theta_{\mathrm{PL}} \sin \left(\Phi-\Phi_{\mathrm{PL}}\right), \\
& \nu(\Theta, \Phi)=\sin \Theta\left(\sin \Theta \cos \Theta_{\mathrm{PL}}-\cos \Theta \sin \Theta_{\mathrm{PL}} \cos \left(\Phi_{\mathrm{PL}}-\Phi\right)\right), \\
& \zeta(\Theta, \Phi)=-\sin \Theta_{\mathrm{PL}} \cos \left(\Phi_{\mathrm{PL}}-\Phi\right), \\
& \beta(\Theta, \Phi)=\sin \Theta_{\mathrm{PL}} \sin \left(\Phi_{\mathrm{PL}}-\Phi\right) .
\end{aligned}
$$

Furthermore, by developing also the energy density $E$ in Taylor series around the equilibrium state in analogy with what was done in (24) for HDMM, we notice that only the second derivatives appear in the equation. Indeed, the inessential constant term can be neglected and the first derivatives vanish at equilibrium. Moreover, considering that the new Lagrangian variables $(\delta \theta, \delta \phi)$ have to exhibit a time dependence proportional to $\exp (\mathrm{i} \omega t)$ (being $\omega / 2 \pi$ the frequency of the spin-wave eigenmode), we end up with

$$
\begin{aligned}
& \delta \phi\left[E_{\phi \phi}+\frac{\sigma J M_{s}}{\gamma} \eta\right]+\delta \theta\left[E_{\phi \theta}+\frac{\sigma J M_{s}}{\gamma} \chi\right] \\
& =\delta \phi\left[-i \omega \frac{\alpha M_{s}}{\gamma} \sin ^{2} \Theta\right]+\delta \theta\left[i \omega \frac{M_{s}}{\gamma} \sin \Theta\right], \\
& \delta \phi\left[E_{\theta \phi}+\frac{\sigma J M_{s}}{\gamma} \zeta\right]+\delta \theta\left[E_{\theta \theta}\right] \\
& \quad=\delta \phi\left[-i \omega \frac{M_{s}}{\gamma} \sin \Theta\right]+\delta \theta\left[-i \omega \frac{\alpha M_{s}}{\gamma}\right],
\end{aligned}
$$

where the subscripts stand for partial derivative with respect to the indicated variables, whose explicit expressions can be found in the Section 2.1. The terms involving $\beta(\Theta, \Phi)$ and $\nu(\Theta, \Phi)$ have been disregarded since they do not exhibit an explicit time dependence.

By setting $\lambda=\gamma / M_{s} \omega$ and $\tilde{\sigma}=\sigma M_{s} / \gamma$, (78) can be recast in the form of a complex generalized non-Hermitian eigenvalue problem

$$
\underline{\underline{A}} \vec{v}=\lambda \underline{\underline{B}} \vec{v}
$$

with

$$
\begin{aligned}
& \underline{\underline{A}}=\left[\begin{array}{cc}
-i \alpha \sin ^{2} \Theta & i \sin \Theta \\
-i \sin \Theta & -i \alpha
\end{array}\right], \\
& \underline{\underline{B}}=\left[\begin{array}{cc}
E_{\phi \phi}+\tilde{\sigma} J \eta & E_{\phi \theta}+\tilde{\sigma} J \chi \\
E_{\theta \phi}+\tilde{\sigma} J \zeta & E_{\theta \theta}
\end{array}\right], \\
& \vec{v}=\left[\begin{array}{ll}
\delta \phi, & \delta \theta
\end{array}\right]^{T} .
\end{aligned}
$$

As expected, if no damping and current are taken into account $\left(\alpha=0, J=0 \mathrm{~A} / \mathrm{m}^{2}\right)$, the system in (79) so obtained recovers exactly the fully conservative HDMM one, where the matrix $\underline{A}$ is Hermitian, whereas the matrix $\underline{B}$ is real and symmetric (see (40)), and all the corresponding eigenvalues are real.

In the presence of dissipative effects due to damping and spin torque, the symmetry of the problem is strongly reduced and the corresponding eigenvalues will be, in general, complex quantities: the real part represents the mode frequency, whereas the imaginary part defines the mode decay rate. With this information in hands, it will be possible to establish, after a single iteration, the subset of the spin-wave normal modes which becomes unstable after the application of a spin-polarized current. In detail, for any applied current below the excitation threshold, the imaginary parts of all eigenvalues have to be positive, recalling the behavior of a damped oscillator. On the contrary, for current values above the excitation threshold, the imaginary parts of the activated normal modes switch to a negative value, giving rise to the instability mechanism which determines, in the time domain, the growth of the precession cone and, in turn, the change of the generated frequency. 
Finally, it should be mentioned that, unlike HDMM, LDMM cannot be recast in a nongeneralized eigenvalue problem form.

3.1.2. LDMM for an Isolated System Composed by N Interacting Magnetic Momenta. Let us consider now the magnetic system of our micromagnetic calculations composed by a finite number $N$ of interacting magnetic momenta. Within our nanoscale numerical approach, each magnetic momentum is identified by means of a micromagnetic cell. The generalization of $(78)$ to $N$ cells $(k=1, \ldots, N)$ leads to a system of $2 \mathrm{~N}$ scalar and linear equations as follows:

$$
\begin{gathered}
\lambda\left\{\sum_{l=1}^{N}\left[E_{\phi_{k} \phi_{l}} \delta \phi_{l}+E_{\phi_{k} \theta_{l}} \delta \theta_{l}\right]+\delta \theta_{k}\left[\tilde{\sigma} J \chi_{k}\right]+\delta \phi_{k}\left[\tilde{\sigma} J \eta_{k}\right]\right\} \\
=\delta \phi_{k}\left[-i \alpha \sin ^{2} \Theta_{k}\right]+\delta \theta_{k}\left[i \sin \Theta_{k}\right], \\
\lambda\left\{\sum_{l=1}^{N}\left[E_{\theta_{k} \phi_{l}} \delta \phi_{l}+E_{\theta_{k} \theta_{l}} \delta \theta_{l}\right]+\delta \phi_{k}\left[\tilde{\sigma} J \zeta_{k}\right]\right\} \\
=\delta \phi_{k}\left[-i \sin \Theta_{k}\right]+\delta \theta_{k}[-i \alpha] .
\end{gathered}
$$

The system in (81) can be analogously formulated as a complex generalized non-Hermitian eigenvalue problem (in the same form as (79)), where

$$
\begin{gathered}
\underline{\underline{A}}=\left[\begin{array}{ccccc}
-i \alpha \sin ^{2} \Theta_{1} & i \sin \Theta_{1} & 0 & 0 & \ldots \\
-i \sin \Theta_{1} & -i \alpha & 0 & 0 & \ldots \\
0 & 0 & -i \alpha \sin ^{2} \Theta_{2} & i \sin \Theta_{1} & \ldots \\
0 & 0 & -i \sin \Theta_{2} & -i \alpha & \ldots \\
\ldots & \cdots & \cdots & \cdots & \cdots
\end{array}\right], \\
\underline{\underline{B}}=\left[\begin{array}{ccccc}
E_{\phi_{1} \phi_{1}}+\tilde{\sigma} J \eta_{1} & E_{\phi_{1} \theta_{1}}+\tilde{\sigma} J \chi_{1} & E_{\phi_{1} \phi_{2}} & E_{\phi_{1} \theta_{2}} & \cdots \\
E_{\theta_{1} \phi_{1}}+\tilde{\sigma} J \zeta_{1} & E_{\theta_{1} \theta_{1}} & E_{\theta_{1} \phi_{2}} & E_{\theta_{1}} \theta_{2} & \ldots \\
E_{\phi_{2} \phi_{1}} & E_{\phi_{2} \theta_{1}} & E_{\phi_{2} \phi_{2}}+\tilde{\sigma} J \eta_{2} & E_{\phi_{2} \theta_{2}}+\tilde{\sigma} J \chi_{2} & \ldots \\
E_{\theta_{2} \phi_{1}} & E_{\theta_{2} \theta_{1}} & E_{\theta_{1} \phi_{1}}+\tilde{\sigma} J \zeta_{1} & E_{\theta_{2} \theta_{2}} & \ldots \\
\cdots & \cdots & \cdots & \cdots & \ldots
\end{array}\right], \\
\vec{v}=\left[\delta \phi_{1}, \delta \theta_{1}, \delta \phi_{2}, \delta \theta_{2}, \ldots \ldots \delta \phi_{N}, \delta \theta_{N}\right]^{T} .
\end{gathered}
$$

It is also interesting to notice that both matrices $\underline{A}$ and $\underline{B}$ appearing in (80) and (82) admit a decomposition which allows to separate the conservative part from the nonconservative one

$$
\begin{aligned}
& \underline{\underline{A}}=\underline{\underline{A_{c}}}+\underline{\underline{A_{n c}}} \\
& \underline{\underline{B}}=\underline{\underline{B_{c}}}+\underline{\underline{B_{n c}}},
\end{aligned}
$$

where

$$
\begin{aligned}
& \underline{\underline{A_{c}}}=\left[\begin{array}{ccccc}
0 & i \sin \Theta_{1} & 0 & 0 & \cdots \\
-i \sin \Theta_{1} & 0 & 0 & 0 & \cdots \\
0 & 0 & 0 & i \sin \Theta_{1} & \ldots \\
0 & 0 & -i \sin \Theta_{2} & 0 & \cdots \\
\cdots & \cdots & \cdots & \cdots & \cdots
\end{array}\right] \text {, } \\
& \underline{\underline{A_{n c}}}=\left[\begin{array}{ccccc}
-i \alpha \sin ^{2} \Theta_{1} & 0 & 0 & 0 & \cdots \\
0 & -i \alpha & 0 & 0 & \cdots \\
0 & 0 & -i \alpha \sin ^{2} \Theta_{2} & 0 & \cdots \\
0 & 0 & 0 & -i \alpha & \cdots \\
\cdots & \cdots & \cdots & \cdots & \cdots
\end{array}\right], \\
& \underline{\underline{B_{c}}}=\left[\begin{array}{ccccc}
E_{\phi_{1} \phi_{1}} & E_{\phi_{1} \theta_{1}} & E_{\phi_{1} \phi_{2}} & E_{\phi_{1} \theta_{2}} & \cdots \\
E_{\theta_{1} \phi_{1}} & E_{\theta_{1} \theta_{1}} & E_{\theta_{1} \phi_{2}} & E_{\theta_{1} \theta_{2}} & \cdots \\
E_{\phi_{2} \phi_{1}} & E_{\phi_{2} \theta_{1}} & E_{\phi_{2} \phi_{2}} & E_{\phi_{2} \theta_{2}} & \cdots \\
E_{\theta_{2} \phi_{1}} & E_{\theta_{2} \theta_{1}} & E_{\theta_{1} \phi_{1}} & E_{\theta_{2} \theta_{2}} & \cdots \\
\cdots & \cdots & \cdots & \cdots & \cdots
\end{array}\right], \\
& \underline{\underline{B_{n c}}}=\left[\begin{array}{ccccc}
\tilde{\sigma} J \eta_{1} & \tilde{\sigma} J \chi_{1} & 0 & 0 & \cdots \\
\tilde{\sigma} J \zeta_{1} & 0 & 0 & 0 & \ldots \\
0 & 0 & \tilde{\sigma} J \eta_{2} & \tilde{\sigma} J \chi_{2} & \ldots \\
0 & 0 & \tilde{\sigma} J \zeta_{1} & 0 & \ldots \\
\cdots & \ldots & \cdots & \ldots & \ldots
\end{array}\right] .
\end{aligned}
$$

The results of this approach have been successfully compared with those coming from another micromagnetic framework which integrates the LLGS equation in the time domain by using a finite-difference scheme $[22,23,35]$. In these works, we evaluated the accuracy of the LDMM approach in determining the excitation threshold and studied in detail the reorientational phase transition which takes place when the direction of the external magnetic field is varied.

Finally, we would like to mention that the extension of the LDMM approach to model the more realistic (and attractive) nonlinear and nonautonomous dynamics [30, 36$41]$ is currently under study.

\section{Acknowledgments}

The research leading to these results has received funding from the European Community's Seventh Framework Programme (FP7/2007-2013) under Grant agreement no. 228673 (MAGNONICS). The authors acknowledge the Project MAT2011-28532-C03-01 from Spanish government.

\section{References}

[1] K. Y. Guslienko and A. N. Slavin, "Spin-waves in cylindrical magnetic dot arrays with in-plane magnetization," Journal of Applied Physics, vol. 87, no. 9, pp. 6337-6339, 2000.

[2] B. A. Ivanov and C. E. Zaspel, "Magnon modes for thin circular vortex-state magnetic dots," Applied Physics Letters, vol. 81, no. 7, pp. 1261-1263, 2002.

[3] C. E. Zaspel, B. A. Ivanov, J. P. Park, and P. A. Crowell, "Excitations in vortex-state permalloy dots," Physical Review B, vol. 72, no. 2, Article ID 024427, 2005.

[4] R. Zivieri and F. Nizzoli, "Theory of spin modes in vortex-state ferromagnetic cylindrical dots," Physical Review B, vol. 71, no. 1, Article ID 014411, 2005. 
[5] R. Zivieri and F. Nizzoli, "Erratum: theory of spin modes in vortex-state ferromagnetic cylindrical dots," Physical Review B, vol. 74, no. 21, Article ID 219901, 2006.

[6] B. A. Ivanov and C. E. Zaspel, "High frequency modes in vortex-state nanomagnets," Physical Review Letters, vol. 94, no. 2, Article ID 027205, 2005.

[7] R. Zivieri and R. L. Stamps, "Theory of spin wave modes in tangentially magnetized thin cylindrical dots: a variational approach," Physical Review B, vol. 73, no. 14, Article ID 144422, 2006.

[8] R. Zivieri, G. Santoro, and A. Franchini, "Localized spin modes in ferromagnetic cylindrical dots with in-plane magnetization," Journal of Physics Condensed Matter, vol. 19, no. 30, Article ID 305012, 2007.

[9] R. Zivieri and F. Nizzoli, "Dipolar magnetic fields of spin excitations in vortex-state cylindrical ferromagnetic dots," Physical Review B, vol. 78, no. 6, Article ID 064418, 2008.

[10] V. Novosad, M. Grimsditch, K. Y. Guslienko, P. Vavassori, Y. Otani, and S. D. Bader, "Spin excitations of magnetic vortices in ferromagnetic nanodots," Physical Review B, vol. 66, no. 5, Article ID 052407, pp. 524071-524074, 2002.

[11] R. Zivieri and F. Nizzoli, "Spin excitations in vortex-state magnetic cylindrical dots: from nanometric to micrometric size," in Electromagnetic, Magnetostatic, and Exchange-Interaction Vortices in Confined Magnetic Structures, E.O. Kamenetskii, Ed., Transworld Research Network, Kerala, India, 2009.

[12] “OOMMF user's guide,” NIST, Gaithersburg, Md, USA.

[13] “LLG micromagnetic simulator,"llgmicro@mindsping.com.

[14] F. Boust and N. Vukadinovic, "Micromagnetic simulations of vortex-state excitations in soft magnetic nanostructures," Physical Review B, vol. 70, no. 17, Article ID 172408, pp. 14, 2004.

[15] M. Grimsditch, L. Giovannini, F. Montoncello, F. Nizzoli, G. K. Leaf, and H. G. Kaper, "Magnetic normal modes in ferromagnetic nanoparticles: a dynamical matrix approach," Physical Review B, vol. 70, no. 5, Article ID 054409, 7 pages, 2004.

[16] L. Giovannini, F. Montoncello, and F. Nizzoli, "Effect of interdot coupling on spin-wave modes in nanoparticle arrays," Physical Review B, vol. 75, no. 2, Article ID 024416, 2007.

[17] R. Zivieri, L. Giovannini, and F. Nizzoli, "Acoustical and optical spin modes of multilayers with ferromagnetic and antiferromagnetic coupling," Physical Review B, vol. 62, no. 22, pp. 14950-14955, 2000.

[18] G. Gubbiotti, G. Carlotti, A. Montecchiari et al., "Brillouin light scattering study of ferromagnetically coupled $\mathrm{Cu} / \mathrm{Fe}(110) / \mathrm{Cu} / \mathrm{Fe}(110) / \mathrm{Cu} / \mathrm{Si}(111)$ heterostructures: Bilinear exchange magnetic coupling," Physical Review B, vol. 62, no. 23, pp. 16109-16115, 2000.

[19] R. Zivieri, F. Montoncello, L. Giovannini et al., "Effect of interdot separation on collective magnonic modes in Chains of rectangular dots," IEEE Transactions on Magnetics, vol. 47, no. 6, pp. 1563-1566, 2011.

[20] S. Tacchi, F. Montoncello, M. Madami et al., "Band diagram of spin waves in a two-dimensional magnonic crystal," Physical Review Letters, vol. 107, no. 12, Article ID 127204, 2011.

[21] R. Zivieri, S. Tacchi, F. Montoncello et al., "Bragg diffraction of spin waves from a two-dimensional antidot lattice," Physical Review B, vol. 85, no. 1, Article ID 012403, 2012.

[22] G. Consolo, G. Gubbiotti, L. Giovannini, and R. Zivieri, "Lagrangian formulation of the linear autonomous magnetization dynamics in spin-torque auto-oscillators," Applied Mathematics and Computation, vol. 217, no. 21, pp. 8204$8215,2011$.
[23] G. Consolo, L. Giovannini, and R. Zivieri, "Excitation of magnetic normal modes by spin-torque: a Lagrangian approach," Journal of Applied Physics, vol. 111, Article ID 07C916, 3 pages, 2012.

[24] F. Montoncello and F. Nizzoli, "Application of the dynamical matrix approach to the investigation of spin excitations in nanometric dots," in Magnetic Properties of Laterally Confined Nanometric Structures, p. 131, Transworld Research Network, Kerala, India, 2006.

[25] L. D. Landau and E. M. Lifshitz, "Theory of the dispersion of magnetic permeability in ferromagnetic bodies," Physik, $Z$. Sowjetunion, vol. 8, p. 153, 1935.

[26] A. Slavin and V. Tiberkevich, "Nonlinear auto-oscillator theory of microwave generation by spin-polarized current," IEEE Transactions on Magnetics, vol. 45, no. 4, pp. 1875-1918, 2009.

[27] T. L. Gilbert, "A phenomenological theory of damping in ferromagnetic materials," IEEE Transactions on Magnetics, vol. 40, no. 6, pp. 3443-3449, 2004.

[28] J. C. Slonczewski, "Current-driven excitation of magnetic multilayers," Journal of Magnetism and Magnetic Materials, vol. 159, no. 1-2, pp. L1-L7, 1996.

[29] J. C. Slonczewski, "Excitation of spin waves by an electric current," Journal of Magnetism and Magnetic Materials, vol. 195, no. 2, pp. L261-L268, 1999.

[30] G. Consolo, B. Azzerboni, G. Gerhart, G. A. Melkov, V. Tiberkevich, and A. N. Slavin, "Excitation of self-localized spin-wave bullets by spin-polarized current in in-plane magnetized magnetic nanocontacts: a micromagnetic study," Physical Review B, vol. 76, no. 14, Article ID 144410, 2007.

[31] G. Consolo, G. Finocchio, L. Torres, M. Carpentieri, L. LopezDiaz, and B. Azzerboni, "Spin-torque switching in $\mathrm{Py} / \mathrm{Cu} / \mathrm{Py}$ and $\mathrm{Py} / \mathrm{Cu} / \mathrm{CoPt}$ spin-valve nanopillars," Journal of Magnetism and Magnetic Materials, vol. 316, no. 2, pp. 492-495, 2007.

[32] S. Bonetti, V. Tiberkevich, G. Consolo et al., "Experimental evidence of self-localized and propagating spin wave modes in obliquely magnetized current-driven nanocontacts," Physical Review Letters, vol. 105, no. 21, Article ID 217204, 2010.

[33] G. Consolo, C. Currò, E. Martinez, and G. Valenti, "Mathematical modeling and numerical simulation of domain wall motion in magnetic nanostrips with crystallographic defects," Applied Mathematical Modelling, vol. 36, no. 10, pp. 48764886, 2012.

[34] G. Consolo, L. Lopez-Diaz, L. Torres, G. Finocchio, A. Romeo, and B. Azzerboni, "Nanocontact spin-transfer oscillators based on perpendicular anisotropy in the free layer," Applied Physics Letters, vol. 91, no. 16, Article ID 162506, 2007.

[35] A. Romeo, G. Finocchio, M. Carpentieri, L. Torres, G. Consolo, and B. Azzerboni, "A numerical solution of the magnetization reversal modeling in a permalloy thin film using fifth order Runge-Kutta method with adaptive step size control," Physica B, vol. 403, no. 2-3, pp. 464-468, 2008.

[36] G. Finocchio, I. Krivorotov, M. Carpentieri et al., "Magnetization dynamics driven by the combined action of ac magnetic field and dc spin-polarized current," Journal of Applied Physics, vol. 99, no. 8, Article ID 08G507, 2006.

[37] G. Consolo, L. Lopez-Diaz, L. Torres, G. Finocchio, A. Romeo, and B. Azzerboni, "Nanocontact spin-transfer oscillators based on perpendicular anisotropy in the free layer," Applied Physics Letters, vol. 91, no. 16, Article ID 162506, 2007.

[38] Y. Zhou, V. Tiberkevich, G. Consolo et al., "Oscillatory transient regime in the forced dynamics of a nonlinear auto oscillator," Physical Review B, vol. 82, no. 1, Article ID 012408, 2010. 
[39] G. Consolo, V. Puliafito, G. Finocchio et al., "Combined frequency-amplitude nonlinear modulation: theory and applications," IEEE Transactions on Magnetics, vol. 46, no. 9, pp. 3629-3634, 2010.

[40] V. Puliafito, G. Consolo, L. Lopez-Diaz, and B. Azzerboni, "Micromagnetic analysis of nonlinear dynamics in spintronic analog modulators," IEEE Transactions on Magnetics, vol. 45, no. 11, pp. 5239-5242, 2009.

[41] G. Consolo and V. Puliafito, "Analytical and micromagnetic study of nonlinear amplitude modulation in spintronic modulators," IEEE Transactions on Magnetics, vol. 46, no. 6, pp. 2063-2066, 2010. 

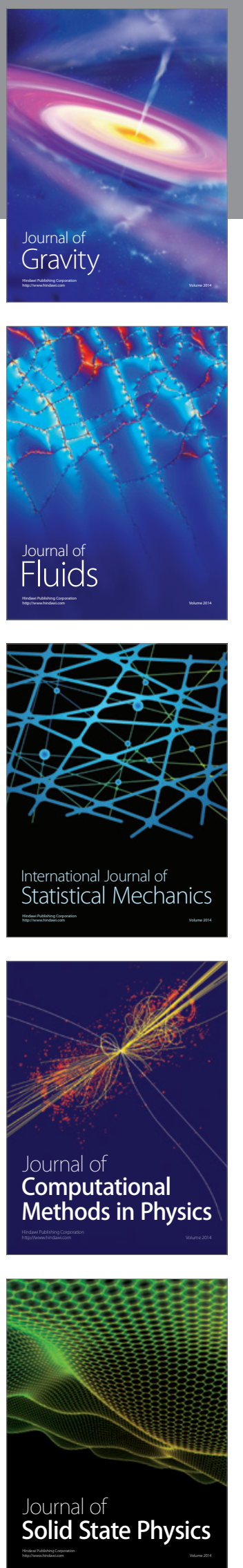

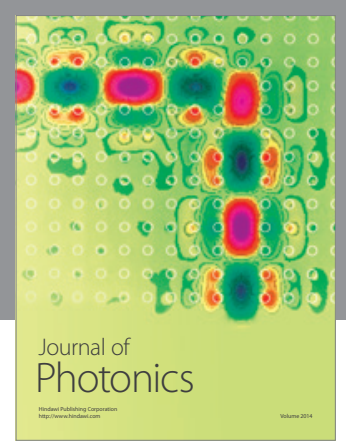

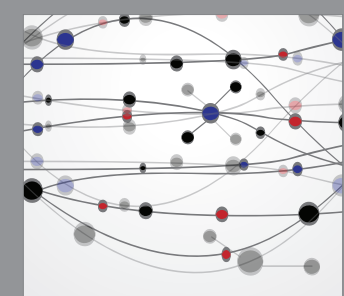

The Scientific World Journal
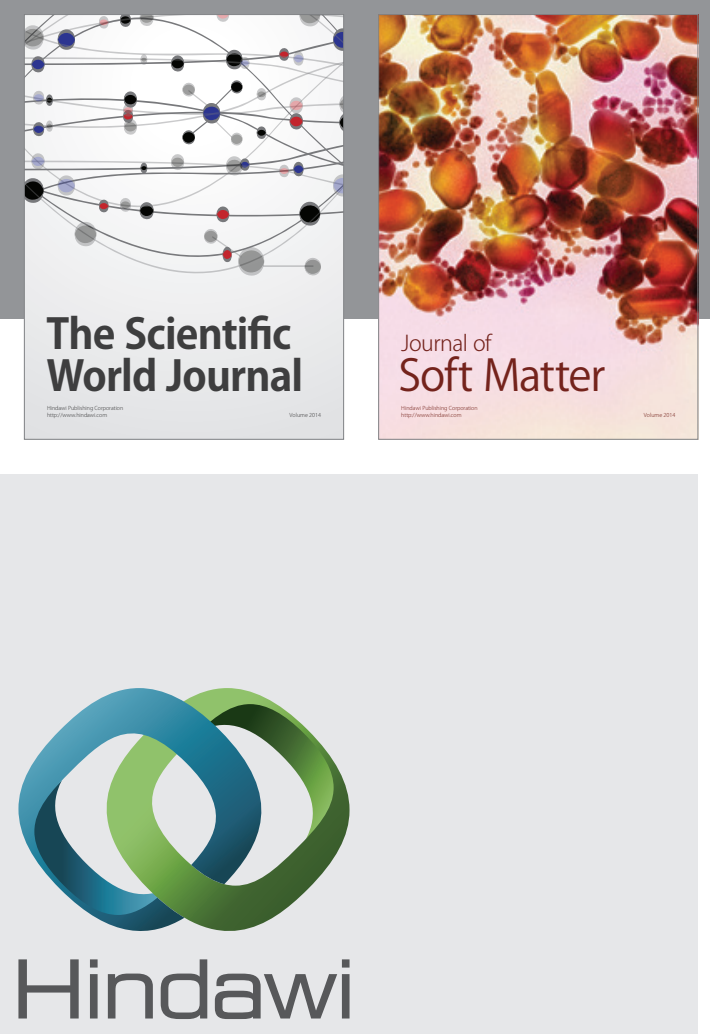

Submit your manuscripts at

http://www.hindawi.com
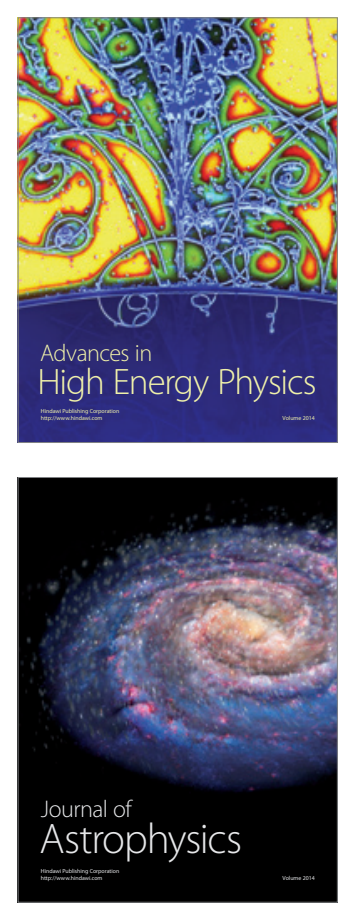
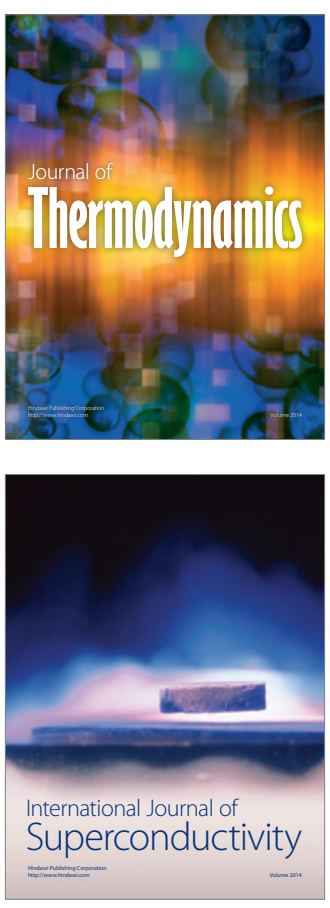
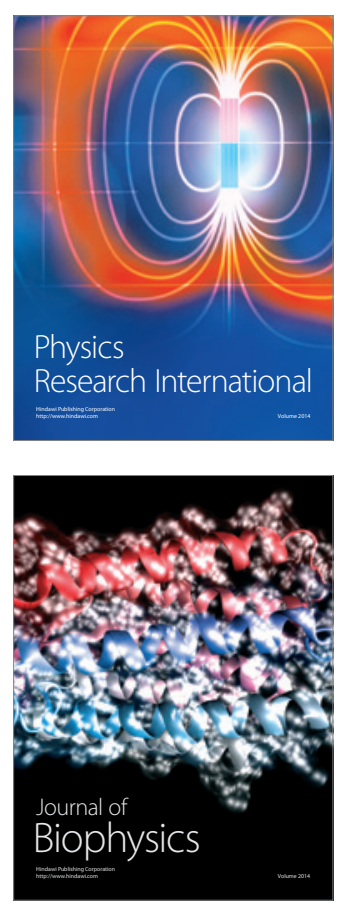
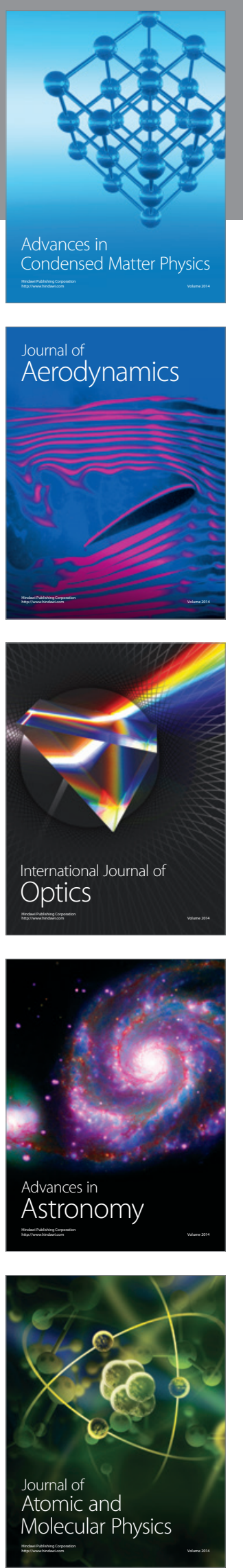